MANUEL BÄRWALD

\title{
DER UMGANG MIT FORSCHUNGSDATEN IN DER HISTORISCHEN MUSIKWISSENSCHAFT
}

Masterarbeit im Rahmen des Weiterbildenden Masterstudiengangs

Bibliotheks- und Informationswissenschaft im Fernstudium

an der Humboldt-Universität zu Berlin

Eingereicht am 9. Mai 2017

Um ein Vorwort ergänzt und veröffentlicht im März 2021

DOI:

http://dx.doi.org/10.25366/2021.06

Informationen zum Autor: $\quad \underline{\text { https://orcid.org/0000-0002-8688-7508 }}$

Dieses Werk steht unter der Lizenz Creative Commons Namensnennung 4.0 International:

https://creativecommons.org/licenses/by/4.0/deed.de 


\section{Vorwort}

Im Oktober 2020 hat die erste Förderphase zum Aufbau einer Nationalen Forschungsdateninfrastruktur (NFDI) für das deutsche Wissenschaftssystem begonnen. Innerhalb der NFDI haben sich die Gesellschaft für Musikforschung und zahlreiche musikwissenschaftliche Forschungsinstitutionen gemeinsam mit Vertretern der Fachbereiche Architektur, Kunstgeschichte, Theater-, Film- und Medienwissenschaft zum Konsortium NFDI4Culture zusammengeschlossen, um Strategien und Werkzeuge für ein fachspezifisches Forschungsdatenmanagement für materielle und immaterielle Kulturgüter zu entwickeln. Für das Fach Musikwissenschaft erfahren die Grundsätze zum Umgang mit Forschungsdaten der Allianz der deutschen Wissenschaftsorganisationen aus dem Jahr 2010 und die Leitlinien zum Umgang mit Forschungsdaten der Deutschen Forschungsgemeinschaft aus dem Jahr 2015 hier zum ersten Mal eine systematische Anwendung.

Die vorliegende Arbeit wurde am 9. Mai 2017 an der Humboldt-Universität zu Berlin als Masterarbeit eingereicht und am 18. September 2017 erfolgreich verteidigt. Aufgrund der vorstehend beschriebenen Entwicklungen haben die hier veröffentlichten Untersuchungsergebnisse und Beobachtungen bis heute grundsätzlich nichts an ihrer Gültigkeit verloren, gleichwohl es mit der NFDI nun aber eine konkrete Perspektive für den Aufbau eines fachspezifischen Forschungsdatenmanagements für das Fach Musikwissenschaft gibt.

Ich bin daher gerne der Einladung gefolgt, die Arbeit auch knapp vier Jahre nach ihrer Entstehung auf musiconn.publish zu veröffentlichen. Der Text spiegelt den Sach- und Wissensstand vom Zeitpunkt seiner Einreichung wieder und wurde für die vorliegende Publikation nur marginal überarbeitet. Verlinkungen wurden geprüft und, wo nötig, aktualisiert. Der letzte Aufruf sämtlicher Links ist am 5. März 2021 erfolgt. Im Literaturverzeichnis wurden die thematisch verwandten Arbeiten von Barbara Alge und Stephan Wünsche ergänzt, die zwischenzeitlich erschienen sind.

Bedanken möchte ich mich an dieser Stelle bei den Betreuerinnen der Arbeit, Vivien Petras und Stephanie van de Sand, für ihre Unterstützung bei der Durchführung des Masterarbeitsvorhabens, sowie bei Barbara Wiermann und Andrea Hammes für die Betreuung des Publikationsprozesses. 


\section{Inhalt}

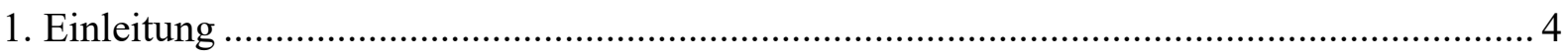

1.1. Digitales Musikwissenschaftliches Arbeiten - eine Positionsbestimmung ..................... 6

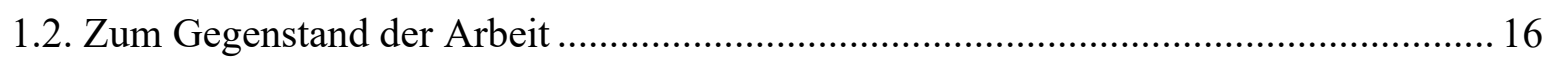

2. Forschungsdaten in der musikwissenschaftlichen Editions- und Quellenarbeit .................. 19

2.1. ,Analoges Forschungsdatenmanagement ${ }^{\star}$ in der Musikedition und der Arbeit mit musikalischen Quellen am Beispiel der Bach-Gesamtausgabe.......................................... 19

2.2. Musikeditorisches und quellengebundenes Arbeiten als ,Small Science‘?.................... 25

3. Der ,Digital Turn“ in der Musikwissenschaft und seine Konsequenzen für den Umgang mit fachspezifischen Forschungsdaten in den Bereichen Musikedition und musikalische

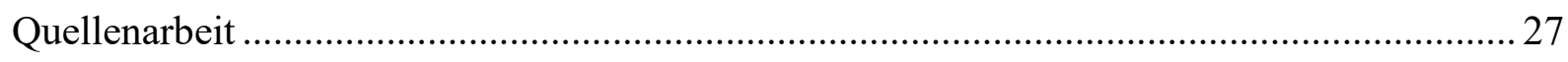

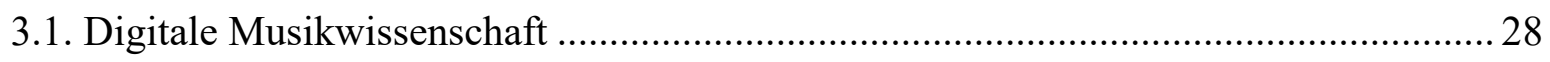

3.2. Digitale Editionsarbeit in der Musikwissenschaft........................................................ 30

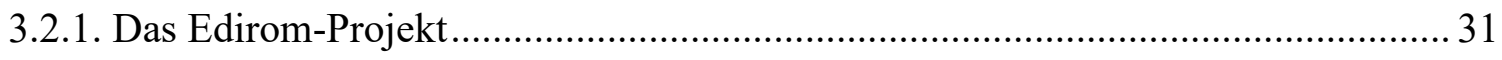

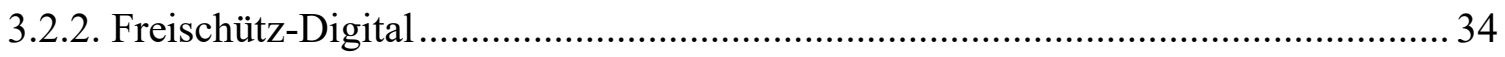

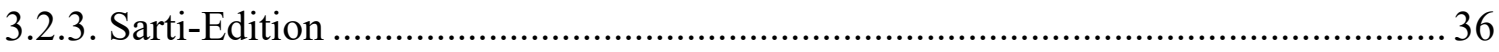

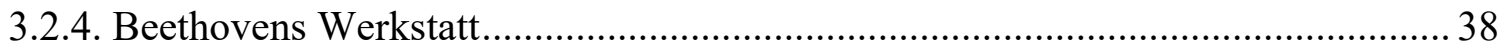

3.3. Digitale Erschließung musikalischer und musikgeschichtlicher Quellen ..................... 43

3.3.1. KoFIM Berlin - Kompetenzzentrum Forschung und Information Musik ............. 44

3.3.2. Sammlung Manfred Gorke - Digitalisierung und Erschließung .............................. 47

3.3.3. Repertoire-Erforschung des Thomanerchores......................................................... 49

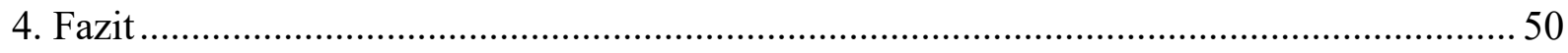

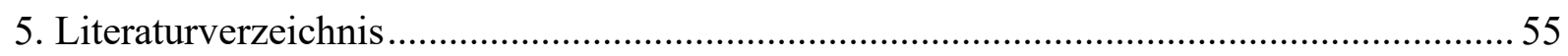




\section{Einleitung}

Mit dem Ausruf der Digitalen Agenda der Bundesregierung und der Berufung des Rats für Informationsinfrastrukturen durch die Gemeinsame Wissenschaftskonferenz von Bund und Ländern hat das Thema Forschungsdatenmanagement in den Wissenschaften seit 2014 die höchste nationale Ebene erreicht und wird von allen Protagonisten mit zunehmender Ernsthaftigkeit diskutiert. Während das Thema in vielen Naturwissenschaften zum Teil seit Jahrzehnten diskutiert wird und erprobte Methoden zur Verwaltung fachspezifischer Messdaten und unpublizierter Forschungsergebnisse zur Verfügung stehen, hatte das Thema in den Geisteswissenschaften lange Zeit keine spezifische Relevanz. Projekt- oder institutionenbezogene Einzellösungen zur Verwaltung und dauerhaften Speicherung nichtpublizierten Wissens waren hier seit Jahrhunderten guter und akzeptierter wissenschaftlicher Standard. Erst im Zuge der allgemeinen Digitalisierung begann eine Transformation der Arbeitsprozesse und -mittel, auf deren Grundlage sich ein Bedarf nach fachspezifischen digitalen Methoden und Werkzeugen zunehmend artikuliert hat.

Entsprechend schreitet die Entwicklung der Digital Humanities in den verschiedenen Fachdisziplinen auch auf ganz unterschiedliche Art und Weise voran. Die Bereitschaft zur Nutzung digitaler Werkzeuge und zur Etablierung digitaler Arbeitstechniken und Workflows ist jeweils sehr unterschiedlich ausgeprägt; genauso heterogen ist das qualitative und quantitative Auftreten von Forschungsfeldern, die digital arbeiten oder sich zumindest digitaler Werkzeuge und Arbeitstechniken bedienen.

Das ist zunächst ein subjektiver Eindruck, der sich unter bestimmten Prämissen allerdings recht gut mit ein paar Fakten belegen lässt: Da die Forschungsarbeit von Wissenschaftlern, die vorrangig digital arbeiten, auch Forschungsdaten produziert, die vorrangig digital vorliegen und entsprechend zu verwalten sind, scheint der Umgang mit diesen Forschungsdaten, ein guter Indikator für die digitale ,Evolutionsstufe ‘ eines Faches zu sein. Messbar - das heißt ablesbar - wird dieser Indikator an verschiedenen Stellen, die sowohl struktureller als auch technischer Art sind. So darf unterstellt werden, dass eine Fachdisziplin, die vorrangig digital arbeitet, sich beispielsweise über ihre Fachgesellschaft(en) frühzeitig auf spezifische Konzepte und Standards verständigt, wie sie die Deutsche Forschungsgemeinschaft mit ihren Leitlinien zum Umgang mit Forschungsdaten ${ }^{1}$ von den

\footnotetext{
${ }^{1}$ Leitlinien zum Umgang mit Forschungsdaten, verabschiedet durch den Senat der Deutschen Forschungsgemeinschaft am 30. September 2015 (http://www.dfg.de/download/pdf/foerderung/antragstellung/forschungsdaten/richtlinien_forschungsdaten.pdf).
} 
Wissenschaftlern einfordert. ${ }^{2}$ Ein Blick auf die von der Deutschen Forschungsgemeinschaft veröffentlichte Liste mit fachspezifischen Empfehlungen zum Umgang mit Forschungsdaten ${ }^{3}$ zeigt, dass die Geisteswissenschaft hier - neben Naturwissenschaften wie der

Biodiversitätsforschung, den Sozial-, Verhaltens- und Wirtschaftswissenschaften und der Psychologie - gleichwertig betrachtet werden, wobei die textbasierte Editionsarbeit der Sprach- und Literaturwissenschaft hier deutlich fokussiert ist. Repräsentativ ist dieser Eindruck mit Blick auf den Beispielcharakter dieser Sammlung von fachspezifischen Empfehlungen freilich nicht.

Eine großflächigere Momentaufnahme ermöglichst ein Blick auf die technische Infrastruktur, indem man sich einen Überblick über die geisteswissenschaftlichen Forschungsdatenrepositorien verschafft, die im Registry of Research Data Repositories ${ }^{4}$ verzeichnet sind. Die folgende Übersicht zeigt für die vom re3data definierten acht geisteswissenschaftlichen Bereiche die Anzahl der vorhandenen Repositorien (Stand: 24. April 2017)..$^{5}$

I. Ancient Cultures

II. History

III. Fine Arts, Music, Theatre and Media Studies

IV. Linguistics

V. Literary Studies

VI. Non-European Languages and Cultures, Social and Cultural Anthropology, Jewish Studies and Religious

Studies
24 Repositorien

46 Repositorien

39 Repositorien

73 Repositorien

11 Repositorien

24 Repositorien

\footnotetext{
${ }^{2}$ Dass weitere Faktoren den disziplinspezifischen Umgang mit Forschungsdaten - zum Teil in noch größerem Ausmaß - beeinflussen, darf freilich nicht übersehen werden. So sind insbesondere Fragen des Datenschutzes hinsichtlich des Umgangs mit personenbezogenen Daten in den Sozialwissenschaften oder die Beachtung der Guten klinische Praxis (GCP) und der Grundsätze der Guten Laborpraxis (GLP) in den Lebenswissenschaften von zentraler Bedeutung. Gerade in den Geisteswissenschaften haben derlei Überlegung aber eine vergleichsweise geringe Bedeutung.

${ }^{3}$ Fachspezifische Empfehlungen zum Umgang mit Forschungsdaten (https://www.dfg.de/foerderung/antrag_gutachter_gremien/antragstellende/nachnutzung_forschungsdaten. ${ }^{4}$ Registry of Research Data Repositories (www.re3data.org).

${ }^{5} \mathrm{Zu}$ beachten ist bei der Übersicht, dass Schnittmengen, die sich dadurch ergeben, dass sich die einzelnen Repositorien nicht immer eindeutig nur einem der acht Bereiche zuordnen lassen, unberücksichtigt bleiben, wodurch zum Teil eine beträchtliche Schieflage in den Zahlen entsteht, derart dass rein fachspezifische Repositorien für einzelne Bereiche gar nicht existieren. So umfasst der Bereich „Fine Arts, Music, Theatre and Media Studies" drei Untersektionen, die zusammen nur einen Teil der 39 Repositorien dieses Bereichs ausmachen: „Art History“ (11 Repositorien), „Musicology“ (5 Repositorien) und „Theatre and Media Studies“ (3 Repositorien). An rein musikwissenschaftlichen Forschungsdatenrepositorien kennt re3data lediglich das Mutopia Project. Free sheet music for everyone.
} 
Dass die Sprachwissenschaften diese Statistik anführen, dürfte vor allem daran liegen, dass die digitale Verwaltung sprachwissenschaftlicher Forschungsdaten - durch den fortgeschrittenen technischen Entwicklungsstand von Programmen zur Text- und Spracherkennung, die ausgezeichnete Datenbasis an Textdokumenten aus den zahlreichen Digitalisierungsprojekten der vergangenen Jahrzehnte beziehungsweise im World Wide Web überhaupt und der langjährigen Etablierung von Auszeichnungssprachen und Semantisierungskonzepten und einer vergleichsweise langen Tradition der Arbeitsweise mit diesen Methoden und Konzepten - inzwischen ein Niveau erreicht hat, das auf einen sehr guten Standardisierungslevel blicken kann und disziplinspezifische Konzepte etabliert hat, die die Initialisierung neuer digitaler Forschungsvorhaben in vielerlei Hinsicht begünstigt. Überall, wo entsprechende Konzepte und Standards fehlen, ist die Aufnahme digitaler Forschungsvorhaben ungleich schwieriger, da auf keine bestehenden Infrastrukturen, aber auch nicht auf die Expertise der Fachkollegen zurückgegriffen werden kann. Von hier aus wirkt das Problem zurück auf die akademische Lehre, wo die fachspezifischen Methoden und Herausforderungen der Digital Humanities schließlich kaum an den wissenschaftlichen Nachwuchs vermittelt werden können.

\subsection{Digitales Musikwissenschaftliches Arbeiten - eine Positionsbestimmung}

Für eine Arbeit, die sich mit dem Umgang mit Forschungsdaten in der Musikwissenschaft beschäftigen will, ist es daher zunächst sinnvoll, das digitale ,Evolutionsniveau“ des Faches ganz allgemein zu umreißen, um zu verstehen, in welchem Umfang welche Art digitaler Daten anfällt. Dabei sollen im Folgenden zwei parallele Ansätze verfolgt werden: Zum einen soll mit Blick auf das Standardwerk zum musikwissenschaftlichen Arbeiten von Nicole Schwindt-Gross, das in seiner Erstauflage 1992 erschienen ist, ${ }^{6}$ und seinem Vergleich mit der völlig neubearbeiteten Ausgabe, die Matthew Gardner und Sara Springfeld 2014 vorgelegt haben, ${ }^{7}$ der digitale Wandel des Faches über einen Zeitraum von gut zwei Jahrzehnten beschrieben werden. Anschließend wird auf Grundlage der Vorlesungsverzeichnisse der musikwissenschaftlichen Institute des deutschsprachigen Raums ein Überblick über die

\footnotetext{
${ }^{6}$ Schwindt-Gross 1992.

${ }^{7}$ Gardner/Springfeld 2014.
} 
Entwicklung in der Vermittlung digitaler Arbeitstechniken in der akademischen Ausbildung während der vergangenen zehn Jahre gegeben.

Nicole Schwindt-Gross hat mit ihrem Lehrbuch Musikwissenschaftliches Arbeiten den ersten „Leitfaden“ vorgelegt, mit dem „demjenigen, der das Gebiet der Musikwissenschaft neu betritt, Pfade durch den Dschungel gewiesen werden“ sollen. ${ }^{8}$ In der Folge hat sich der Band als Standardwerk der musikwissenschaftlichen Ausbildung etabliert; es dürfte seitdem kaum einen Studierenden des Fachs gegeben haben, der ihn während seiner Einführungsseminare nicht regelmäßig in der Hand gehabt hat. Entlang der Spezifika musikwissenschaftlicher Methoden, Nachschlagewerke und Arbeitswerkzeuge gibt Schwindt-Gross eine klassische Einführung in die analoge wissenschaftliche Arbeitsweise: Recherche und Beschaffung von Quellen und der Umgang mit ihnen, Bibliographieren und Bibliothekskunde und eine Zusammenfassung der Regeln zur wissenschaftlichen Manuskriptgestaltung. Der „Einsatz von EDV“ gilt damals noch als ein Themenfeld, das ,im großen und ganzen für alle Fächer gleich" gilt. $^{9}$

Die Neuausgabe von Gardner und Springfeld nimmt hier 2014 keine grundlegende Neubewertung vor. Ein dem Band vorangestelltes Begleitwort von Nicole Schwindt betont stattdessen gleich zu Beginn, dass, ,auch die virtuellen Ressourcen und das elektronische Arbeitsmanagement" denselben handwerklichen Grundregeln folgt wie der Umgang mit analogen Speichermedien und Quellen: ${ }^{10}$
„Um einen Gegenstand zu untersuchen, muss er zuerst lokalisiert werden. Um Kontexte herzustellen, müssen diese und das Wissen darüber aufgerufen werden. Um etwas sinnvoll zu kommunizieren, bedarf es der Ordnung der Gedanken und der Kanäle, in denen die Einsichten verständlich transportiert werden. Das alles ist wissenschaftliches Handwerk.“

Bei diesem Verständnis des wissenschaftlichen Arbeitsprozesses fällt auf, dass der Schritt der Kontextualisierung und des Abrufs von weiterem Kontextwissen keine physische Komponente zu haben scheint, sondern im Verständnis des Wissenschaftlers rein mental abläuft. Die Verwaltung des kontextualisierten Wissens, das im Vorfeld der

\footnotetext{
${ }^{8}$ Schwindt-Gross 1992, S. 13.

${ }^{9}$ Ebenda, S. 15.

${ }^{10}$ Dies und das Folgende nach Gardner/Springfeld 2014, S. 9.
} 
Wissenskommunikation entsteht, spielt keine Rolle. Forschungsdatenmanagement scheint hiervon ausgehend - kein klassischer Baustein musikwissenschaftlicher Arbeitsweise zu sein. Diesem Gedanken folgen dann auch die Autoren in ihrem Vorwort weitgehend, auch wenn sie schreiben, dass sich seit Erscheinen der Erstausgabe „die organisatorischen und vor allem die technischen Rahmenbedingungen des wissenschaftlichen Arbeitens in vielfältiger Weise verändert haben“": 11

„Die Techniken und Errungenschaften des digitalen Zeitalters haben mittlerweile in fast alle musikwissenschaftlichen Arbeitsbereiche Einzug gehalten - von der Verfügbarkeit reproduzierter Quellen im World Wide Web über neuartige Rechercheund Literaturbeschaffungsmethoden bis hin zum EDV-gestützten Arbeiten mit den Quellen.“

Als ,zentrale Arbeitsgrundlage für den Musikhistoriker“ beschreiben Gardner und Springfeld die historischen Quellen, deren Auswertung in der wissenschaftlichen Fachliteratur vollzogen wird. Die genannten fünf Schritte wissenschaftlichen Arbeitens (Fragestellung definieren, Literatur- und Quellenrecherche, Materialsichtung, Materialbewertung, Darstellung) ${ }^{12}$ entsprechen dem klassischen, bereits von Schwindt-Gross 1992 verwendeten Modell. ${ }^{13}$ Auch wenn es damit strukturell offenbar kein Phänomen wie eine „Digitale Musikwissenschaft ${ }^{* 14}$ zu berücksichtigen gilt, existieren musikwissenschaftsspezifische digitale Arbeitsumgebungen und Services, die Gardner und Springfeld beschreiben.

1. Notenausgaben liegen digital in unterschiedlichen Qualitäten vor: Erstens kann es sich um digitalisierte Werkausgaben handeln, die ursprünglich analog entstanden sind. Hierzu zählt etwa die Neue Mozart-Ausgabe oder ein Großteil der über die Petrucci Music Library abrufbaren Digitalisate gemeinfreier Noten. Daneben entstehen seit einigen Jahren auch born-digital Editionen, die die multimedialen Möglichkeiten des digitalen Arbeitens gezielt einsetzen, um Lesartenvergleiche statt in den kritischen Bericht nun direkt in die Edition einzubinden. Für die digitale Editionsarbeit hat sich die Software Edirom inzwischen als fachspezifischer Quasistandard entwickelt. ${ }^{15}$

\footnotetext{
${ }^{11}$ Dies und das Folgende nach Gardner/Springfeld 2014, S. 11.

12 Ebenda, S. 24-26.

${ }^{13}$ Schwindt-Gross 1992, S. 25-28.

${ }^{14} \mathrm{Zu}$ meiner Verwendung des Begriffs siehe die Ausführungen in Kapitel 3.1. dieser Arbeit.

${ }^{15}$ Gardner/Springfeld 2014, S. $79 f$.
} 
Darüber hinaus kommen auf Anwenderebene in der musikwissenschaftlichen Arbeit schon seit Jahren klassische Notensatzprogramme wie Finale, Sibelius oder Capella zum Einsatz, die die Erstellung eines digitalen Notensatzes erlauben.

2. Die Digitalen Sammlungen insbesondere der großen Bibliotheken mit einem umfangreichen historischen Musikhandschriftenbestand (vor allem Bayerische Staatsbibliothek, Staatsbibliothek zu Berlin (SBB), Sächsische Landesbibliothek Staats- und Universitätsbibliothek Dresden (SLUB), British Library und Bibliothèque Nationale de France) erlauben heute eine immer größer werdende Anzahl ihrer Manuskriptbestände nicht nur online zu recherchieren, sondern sie unmittelbar digital abzurufen. ${ }^{16}$ Eine weitere Säule des Digitalisierungsprozesses von Musikhandschriften sind institutionsübergreifende Digitalisierungsprojekte, bei denen umfangreiche Handschriftenbestände zur Werküberlieferung eines Komponisten digital erschlossen werden. Hier ist insbesondere Bach digital zu nennen. In dem von der SBB, der SLUB und dem Bach-Archiv Leipzig gemeinschaftlich durchgeführten Projekt stehen heute nahezu sämtliche noch erhaltene Bach-Autographe und originale Aufführungsmaterialien sowie Bachs Abschriften fremder Werke kostenfrei online. ${ }^{17}$

3. Für die Recherche musikalischer Quellenbestände ist das RISM. Répertoire International des Sources Musicales, das Internationale Quellenlexikon der Musik, das wichtigste musikwissenschaftliche Werkzeug. Der Katalog dient als Nachweis für musikalische Einzeldrucke vor 1800 (Serie A/I), Musikhandschriften nach 1600 (Serie A/II) und Sammelwerke vom 11. bis 18. Jahrhundert (Serie B). Die Serien A/I und A/II sind über das Portal https://opac.rism.info online und frei zugängig. Serie B liegt als gedruckter Katalog vor.

4. Die beiden großen Musikenzyklopädien, der deutschsprachigen Musik in Geschichte und Gegenwart und dem englischsprachigen New Grove Dictionary of Music and Musicians,${ }^{18}$ wurden inzwischen in digitale Services überführt. Mit den Plattformen GMO und MGG Online liegen die Inhalte von Grove ${ }^{2}$ und $\mathrm{MGG}^{2}$ inzwischen in kostenpflichtigen Onlinefassungen vor. Bei beiden Nachschlagewerken werden die digitalen Inhalte - insbesondere die Literarturverzeichnisse - im Sinne einer dynamischen Enzyklopädie regelmäßig aktualisiert. ${ }^{19}$

\footnotetext{
${ }^{16}$ Ebenda, S. 204.

${ }^{17}$ Ebenda; sowie Bach digital. Geschichte (https://www.bachdigital.de/content/history.xml?XSL.lastPage.SESSION=/content/history.xml).

${ }^{18} \mathrm{MGG}, \mathrm{MGG}^{2}$, Grove und Grove ${ }^{2}$.

${ }^{19}$ Gardner/Springfeld 2014, S. 173; sowie www.mgg-online.com.
} 
5. Analog zu den beiden Musikenzyklopädien in deutscher und englischer Sprache existieren mit RILM und BMS zwei musikwissenschaftliche Fachbibliographien, die einerseits kostenpflichtig (RILM) und andererseits frei (BMS) digital verfügbar sind. Beide Systeme ermöglichen neben der Recherche von selbstständigen Publikationen wie Monographien und Aufsatzsammlungen auch die Suche nach unselbstständigen Veröffentlichungen und liefern kurze englisch- (RILM) beziehungsweise deutschsprachige (BMS) Abstracts zu den Publikationen.

6. Für die Recherche von Bildquellen, die ,in irgendeiner Form mit Musik in Verbindung stehen“, hat sich die freie Online-Datenbank RIdIM. Répertoire International d'Iconographie Musicale als Standard etabliert, auch wenn der Aufbau „noch lange nicht abgeschlossen“ ist. ${ }^{20}$

7. Eine spezialisierte Bilddatenbank für Musikinstrumente - und zugleich eine der neuesten und fortschrittlichsten Errungenschaften in der Digitalisierung des musikwissenschaftlichen Wissens - ist die kostenfreie Datenbank Musical Instrument Museums Online (MIMO). Musikinstrumentensammlungen aus aller Welt beteiligen sich hier an der Onlinestellung ihrer Bestände mit Abbildungen und Objektbeschreibungen, die einheitlichen Standards folgen. ${ }^{21}$

Mit Blick auf die oben genannten fünf Schritte wissenschaftlichen Arbeitens (Fragestellung definieren, Literatur- und Quellenrecherche, Materialsichtung, Materialbewertung, Darstellung) zeigt sich, dass die in der musikwissenschaftlichen Arbeit derzeit verbreitetsten digitalen Arbeitsumgebungen und Services mehr oder weniger ausschließlich den Bereich der Literatur- und Quellenrecherche betreffen und hier zum Teil erhebliche Arbeitserleichterungen bieten; jeder der sieben Punkte lässt sich diesem Bereich zuordnen. Die Themen Editionsarbeit (1), Quellenbeschreibungen in digitalen Sammlungen und Handschriftendatenbanken (2), der Informationsaufbereitung in RISM (3) und der Präsentation von Bildquellen und deren Beschreibung (6 und 7) wirken außerdem in den Arbeitsbereich der Darstellung. Hier wurden neue digitale Systeme zur Präsentation musikwissenschaftlichen Wissens geschaffen, die gegenüber den klassischen analogen Publikationsmöglichkeiten einen erheblichen Mehrwert besitzen. Disziplinspezifische digitale Arbeitsumgebungen und Werkzeuge, die erweiterte Möglichkeiten für die Arbeitsbereiche Materialsichtung, -aufbereitung und -bewertung bieten, scheint es hingegen nicht zu geben,

\footnotetext{
${ }^{20}$ Gardner/Springfeld 2014, S. 212.

${ }^{21}$ Ebenda, S. $214 \mathrm{f}$.
} 
auch wenn auf der Hand liegt, dass die digitale Transformation letztlich alle Schritte des wissenschaftlichen Arbeitens erfassen muss, um Medienbrüche zu vermeiden.

Nach diesem Blick auf die grundlegenden Phänomene des digitalen Wandels im musikwissenschaftlichen Arbeiten soll nun geschaut werden, wie sich diese Veränderung in der akademischen Lehre des Faches widerspiegeln. Dazu erfolgt eine Auswertung der Vorlesungsverzeichnisse der musikwissenschaftlichen Institute des deutschsprachigen Raums auf Grundlage der Zusammenstellung, die die Gesellschaft für Musikforschung auf ihrer Homepage bietet. $^{22}$ Da eine vollständige Auswertung der Verzeichnisse an dieser Stelle zu weit gehen würde, fokussiere ich die Betrachtung auf insgesamt vier zusammenhängende Zeiträume, die jeweils ein Studienjahr umfassen: WS 2007/2008 und SS 2008, WS 2010/2011 und SS 2011, WS 2013/2014 und SS 2014, WS 2016/2017 und SS 2017. Die Auswahl der hier berücksichtigten Lehrveranstaltungen erfolgte qualitativ anhand der Seminartitel, derart dass jene Lehrveranstaltungen in die quantitative Auswertung einflossen, deren Titel eine inhaltliche Ausrichtung auf ,digitale“ oder „,computergestützte“ Arbeitsweisen und Techniken behaupten. ${ }^{23}$ Insgesamt entsprechen 53 Lehrveranstaltungen diesen Voraussetzungen; auf die vier Untersuchungszeiträume (UZ) verteilen sie sich quantitativ wie folgt:

$\begin{array}{lll}\text { UZ } 1 & \text { WS 2007/2008 und SS 2008 } & 7(4+3) \\ \text { UZ 2 } & \text { WS 2010/2011 und SS 2011 } & 14(7+7) \\ \text { UZ 3 } & \text { WS 2013/2014 und SS 2014 } & 9(5+4) \\ \text { UZ 4 } & \text { WS 2016/2017 und SS 2017 } & 22(8+14)\end{array}$

Qualitativ lassen sich die Lehrveranstaltungen vier Bereichen zuordnen: 1. Digitales Arbeiten in der Musikwissenschaft - 2. Digitaler Notensatz und digitale Musikedition 3. Computergestützte Musikanalyse -4 . Sonstiges und spezielle Themen

\footnotetext{
${ }^{22} \mathrm{http}$ ://www.musikforschung.de/index.php/studium-musikwissenschaft/vergangene-lehrveranstaltungen.

${ }^{23}$ Unberücksichtigt bleiben Lehrveranstaltungen zu Themen aus dem Bereich Computermusik und Elektroakustik, da hier der technische Aspekt derart genuin im Vordergrund des Themas steht, dass eine Betrachtung aus dem Blickwinkel der Digital Humanities nicht sinnvoll ist.
} 


\begin{tabular}{|c|c|c|c|c|}
\hline & Bereich 1 & Bereich 2 & Bereich 3 & Bereich 4 \\
\hline UZ 1 & 2 & 3 & 1 & 1 \\
\hline UZ 2 & 4 & 7 & 1 & 2 \\
\hline UZ 3 & 2 & 0 & 6 & 1 \\
\hline UZ 4 & 8 & 9 & 4 & 1 \\
\hline Gesamt & 16 & 19 & 12 & 5 \\
\hline
\end{tabular}

Zwar ist die Abgrenzung der vier Bereiche nicht immer konsistent, dennoch erlaubt sie einen ganz guten Überblick über die Heterogenität des digitalen musikwissenschaftlichen Arbeitens. Die Ausbildung in den Lehrveranstaltungen aus Bereich 1 (Digitales Arbeiten in der Musikwissenschaft) umfasst Themen wie die Rolle der Musik und der Musikwissenschaft im digitalen Zeitalter, ${ }^{24}$ digitale Quellenkunde, ${ }^{25}$ Einführungsveranstaltungen zum computergestützten Arbeiten in der Musikwissenschaft ${ }^{26}$ und Seminare mit Spezialthemen, die aber Einführungscharakter zu digitalen Arbeitsmethoden haben sollen. ${ }^{27}$ Die 16 Lehrveranstaltungen fanden an zehn verschiedenen Institutionen statt, wobei insgesamt ein Viertel allein auf das musikwissenschaftliche Seminar Detmold/Paderborn entfällt. Hier ist das vom Bundesministerium für Bildung und Forschung geförderte Zentrum Musik - Edition - Medien (Zen $|\mathrm{M}| \mathrm{E} \mid \mathrm{M})$ angesiedelt, das sich der Erforschung der „Veränderungen und neuen Möglichkeiten beim Übergang von analogen zu digitalen Musik- und Medieneditionen“

\footnotetext{
${ }^{24}$ Hartmut Hein, Digital Humanities in der Musikwissenschaft, Universität zu Köln, Sommersemester 2017; sowie Julian Caskel, Musik und Digitalisierung, Hochschule für Musik und Tanz Köln, Sommersemester 2017; sowie Andreas Münzmay, Musik und Wissenschaft im digitalen Zeitalter, Universität Paderborn (Musikwissenschaftlichen Seminar Detmold/Paderborn), Wintersemester 2016/17.

${ }_{25}$ Jonathan Gammert, Quellengebundenes Arbeiten in digitalen Zeiten, Johannes Gutenberg Universität Mainz, Sommersemester 2017; sowie Veronika Keller, Mit digitalen Quellen arbeiten, Ludwig-Maximilian-Universität München, Sommersemester 2017; sowie Therese Bruggisser-Lanker, Musikhandschriften original \& digital, Universität Zürich, Sommersemester 2017; sowie Lars-Christian Koch, Kulturgut digital. Das Projekt MIMO „,Musical Instrument Museums Online“, Universität zu Köln, Wintersemester 2010/11 und Sommersemester 2011.

${ }^{26}$ Joachim Iffland, Digitale Präsentation, Hochschule für Musik Detmold (Musikwissenschaftlichen Seminar Detmold/Paderborn), Sommersemester 2017 (im Sommersemester 2014 unter der Seminarleitung von Nikolaos Beer und im Sommersemester 2011 unter der Seminarleitung von Julian Dabbert); sowie Rainer Bayreuther, Digitale Lehr- und Lernumgebungen an der Musikhochschule Trossingen, Wintersemester 2016/17; sowie Bettina Berlinghoff-Eichler, Computergestütztes Arbeiten in der Musikwissenschaft, Universität Regensburg; sowie Christoph Meixner, Einführung in computerunterstütztes Arbeiten in der Musikwissenschaft, Hochschule für Musik Franz Liszt Weimar, Sommersemester 2008.

${ }_{27}$ Jonathan Gammert, Mittelrheinische Musik digital, Johannes Gutenberg Universität Mainz, Sommersemester 2014 (Der Einführungscharakter ergibt sich aus dem Kommentar zur Lehrveranstaltung, der auf der Institutshomepage unter http://www.musikwissenschaft.unimainz.de/musikwissenschaft/veranstaltungen/ss14.htm zuletzt am 24. April 2017 abgerufen wurde und inzwischen nicht mehr erreichbar ist); sowie Lars-Christian Koch, iPod\&Co. Digitale Archive und der Umgang mit Musik, Universität zu Köln, Wintersemester 2007/08.
} 
widmet. ${ }^{28}$ Und hier wurde am 1. August 2016 die bundesweit erste Professur für Digitale Musikedition und Digital Humanities mit Andreas Münzmay besetzt. ${ }^{29}$

Entsprechend wäre zu erwarten, dass sich die besondere Rolle des musikwissenschaftlichen Seminars Detmold/Paderborn gerade auch in Bereich 2 (Digitaler Notensatz und digitale Musikedition) fortsetzt; hier ist das Institut aber mit nur einer Lehrveranstaltung vertreten, die sich im Wintersemester 2016/17 dem Thema Digitale Editionspraxis gewidmet hat. Diese scheinbare Schieflage kommt durch Auswahl der Untersuchungszeiträume zustande und spiegelt nicht den Sachstand wider. Tatsächlich finden am musikwissenschaftlichen Seminar Detmold/Paderborn spätestens seit dem Sommersemester 2014 regelmäßig

Lehrveranstaltungen zum Thema Digitale Musikedition statt. Die Profilierung des Instituts zu der auch die jährlichen Edirom-Summer-Schools ${ }^{30}$ gehören - ist eng verknüpft mit der Person Joachim Veits, der als Editionsleiter der Carl-Maria-von-Weber-Gesamtausgabe und Initiator des Zen $|\mathrm{M}| \mathrm{E} \mid \mathrm{M}$ über jahrelange Erfahrungen in der digitalen Musikedition verfügt. 13 weitere Seminare, die sich ganz allgemein dem Thema digitale Musikedition, Computernotensatz u.ä. gewidmet haben, fanden an den Universitäten in Bayreuth (dreimal), Bremen (dreimal), Frankfurt, Karlsruhe, Kiel, Mainz, Regensburg (zweimal) und Wien statt. Auf einzelne Anwendungen und Standards fokussierte Seminare wurden zu den Themen „Digitale Musikedition mit MEI“,31 „Notensatz mit Finale 2014“32 und „Einführung in die Digitale Musik-Codierung und -Edition mit MEI“33 angeboten. Schon über die Grenzen eines reinen Editionsseminars hinaus gehen die Lehrveranstaltungen von Wolfgang Horn zum Thema „Musikdrucke des 16.-18. Jahrhunderts: vom Digitalisat zur editorischen Erschließung “34 und von Panja Mücke „Editorische Praxis vom frühen Musikdruck bis zur digitalen Werkausgabe“. 35

Die zwölf Lehrveranstaltungen in Bereich 3 (Computergestützte Musikanalyse) fanden an acht verschiedenen Instituten statt. Auch bei diesem Spezialthema digitalen musikwissenschaftlichen Arbeitens zeichnet sich mit Halle/Saale ein Zentrum ab, an dem ein Viertel der Lehrveranstaltungen stattfanden - Grund ist, dass durch Wolfgang Auhagen und

\footnotetext{
${ }^{28}$ Zentrum Musik - Edition - Medien (Zen $\left.|M| E \mid M\right)$. Projektbeschreibung (http://zenmem.de).

${ }^{29}$ Nachrichten aus der Akademie vom 19. August 2016: Prof. Dr. Andreas Münzmay tritt neue AkademieProfessur in der Musikwissenschaft an (http://www.adwmainz.de/nachrichten/artikel/prof-dr-andreasmuenzmay-tritt-neue-akademie-professur-in-der-musikwissenschaft-an.html).

${ }^{30}$ Die Edirom-Summer-School wird am Musikwissenschaftlichen Seminar Detmold/Paderborn in Kooperation mit DARIAH-DE organisiert (http://ess.uni-paderborn.de/about.html).

${ }^{31}$ Robert Klugseder, Universität Wien, Sommersemester 2017.

32 David Witsch, Universität Bonn, Sommersemester 2017.

${ }^{33}$ Stefan Morent, Universität Tübingen, Wintersemester 2016/17.

${ }^{34}$ Universität Regensburg, Wintersemester 2016/17

${ }^{35}$ Universität Münster, Sommersemester 2011.
} 
das Modul „Einsatz von Computerprogrammen zur Musikanalyse“ dieses Thema fest im Curriculum des Instituts verankert ist. Angeboten werden Seminare zu diesem Bereich auch an den Universitäten in Bremen, ${ }^{36}$ Berlin (Humboldt), ${ }^{37}$ Hamburg, ${ }^{38}$ Mainz, ${ }^{39}$ Gießen ${ }^{40}$ sowie an den Musikhochschulen in Leipzig ${ }^{41}$ und Weimar. ${ }^{42}$

Sechs Lehrveranstaltungen, die sich digitalen Themen widmen, ließen sich keinem der drei Bereiche zuordnen, sie sind ihnen aber zumindest teilweise verwandt. So behandelte Stefanie Alisch im Sommersemester 2014 an der Universität Oldenburg mit dem Thema „Ethnologische Forschung in der Zeit der Digitalisierung“ einen Komplex, der vom Ansatz her dem Bereich 1 nahesteht. Die Seminare „Computergestütztes Komponieren mit SuperCollider“, 43 „Computergestützte Stimmanalyse““44 und „Musikalische Interpretation mit dem Computer durchleuchtet: Quantitative Methoden zur computergestützten PerformanceForschung ${ }^{645}$ sind eher den Themen aus Bereich 3 verwandt. Keinem der drei Bereiche nahestehend ist die Lehrveranstaltungen „Mapping Visual Kei. Die digitale Verortung japanischer Popularmusik in Europa“" von Oliver Seibt. ${ }^{46}$

Durch die Begrenzung des Untersuchungszeitraums auf vier Zeitfenster besitzen die quantitativen Schlussfolgerungen aus der Untersuchung der Lehrveranstaltungen mit Themen zum digitalen Arbeiten in der Musikwissenschaft zwar nur eine eingeschränkte Aussagekraft. Dennoch lassen sich einige wesentliche Tendenzen ablesen:

1. Die Gesamtzahl der musikwissenschaftlichen Lehrveranstaltungen zu Themen des digitalen wissenschaftlichen Arbeitens hat an den deutschsprachigen Universitäten in Deutschland, Österreich und der Schweiz in den vergangenen zehn Jahren deutlich zugenommen. Im Vergleich zu Untersuchungszeitraum 1 (WS 2007/2008 und SS 2008) fanden in Untersuchungszeitraum 4 (WS 2016/2017 und SS 2017) dreimal so viele entsprechende Lehrveranstaltungen statt.

2. Es konnten drei große Themenbereiche identifiziert werden, mit denen sich diese Lehrveranstaltungen beschäftigen: „Digitales Arbeiten in der Musikwissenschaft“,

\footnotetext{
${ }^{36}$ Axel Schröter, Sommersemester 2017.

${ }^{37}$ Marcello Lussana, Sommersemester 2017.

${ }^{38}$ Klaus Frieler, Wintersemester 2007/08.

${ }^{39}$ Albert Gräf, Sommersemester 2017.

${ }^{40}$ Ralf von Appen, Wintersemester 2013/14.

${ }^{41}$ Egor Poliakov, Wintersemester 2013/14 und Sommersemester 2014.

42 Martin Pfleiderer, Wintersemester 2013/14.

${ }^{43}$ Johannes Quint, Hochschule für Musik und Darstellende Kunst Frankfurt am Main, Sommersemester 2017.

${ }^{44}$ Josef Pilaj, Kunstuniversität Graz, Wintersemester 2007/08.

${ }^{45}$ Werner Goebl, Universität Wien, Wintersemester 2010/11.

${ }^{46}$ Universität Heidelberg, Sommersemester 2011.
} 
„Digitaler Notensatz und digitale Musikedition“ sowie „Computergestützte Musikanalyse“. Am stärksten vertreten sind Lehrveranstaltungen aus Themenbereich 2, was sicher daran liegt, dass hier die größte Praxisrelevanz in der musikwissenschaftlichen Arbeitswelt für die Studierenden gesehen wird. Themenbereich 1, der sich überblickshaft der Musikwissenschaft in den Digital Humanities und der Einführung in das digitale musikwissenschaftliche Arbeiten widmet, folgt zwar nur auf Platz 2, jedoch mit so geringem Abstand, dass dies mit Blick auf die oben beschriebenen Einschränkungen der Aussagekraft dieser Untersuchung keine statistische Relevanz haben dürfte.

3. Die Lehrveranstaltungen in den drei beschriebenen Themenbereichen haben sich von Untersuchungszeitraum $1 \mathrm{zu}$ Untersuchungszeitraum 4 in einem etwa gleichmäßig proportionalen Verhältnis zueinander entwickelt. ${ }^{47}$

Untersuchungszeitraum $1 \quad 2,0: 3,0: 1,0$

Untersuchungszeitraum $4 \quad 2,0: 2,3: 1,0$

Gesamtverhältnis $\quad 2,0: 2,4: 1,5$

In jedem der drei Bereiche hat sich die Anzahl der Lehrveranstaltungen verdrei- bis vervierfacht.

4. Es lohnt überdies ein quantitativer Blick auf die oben (S. 9) herausgearbeiteten Themenkomplexe in Bereich 1: Zur Rolle der Musik und der Musikwissenschaft im digitalen Zeitalter fanden in den Untersuchungszeiträumen drei Lehrveranstaltungen statt, fünf Seminare oder Übungen widmeten sich der digitalen Quellenkunde und es gab sechs Einführungsveranstaltungen zum computergestützten Arbeiten in der Musikwissenschaft. Auch diese Zahlen zeigen die sehr praxisnahe Verankerung digitaler Themen in den Studienplänen. Digitale Quellenkunde, die Einführung in computergestützte Arbeitstechniken und die beiden Seminare mit ebenfalls stark praxisgeleiteten Spezialthemen machen zusammen über 80 Prozent der Lehrveranstaltungen ${ }^{48}$ in Bereich 1 aus.

5. Das alles sagt aber noch nichts darüber aus, wie stark digitale Inhalte und Arbeitsweisen insgesamt in den Studienplänen der deutschsprachigen Musikwissenschaft verankert sind. Die Gesellschaft für Musikforschung listet auf ihrer Homepage 70 musikwissenschaftliche Institute in Deutschland, Österreich und

\footnotetext{
${ }^{47}$ Die folgenden Zahlen wurden auf eine Nachkommastelle gerundet.

${ }^{48} 13$ (digitale Quellenkunde: 5, Einführung in computergestützte Arbeitstechniken: 6, praxisnahe Spezialthemenseminare: 2) von insgesamt 16 Lehrveranstaltungen in Bereich 1.
} 
der Schweiz. In den drei Untersuchungszeiträumen fanden die 53 Lehrveranstaltungen zu digitalen Themen an 28 Instituten (= 40 Prozent) statt. Auch wenn diese Zahl bei einer vollständigen Betrachtung der Lehrveranstaltungen der vergangenen zehn Jahre sicher ein ganzes Stück höher läge, wird deutlich, dass die musikwissenschaftliche Ausbildung von einer flächendeckenden Vermittlung von digitalen Inhalten und Arbeitsmethoden noch weit entfernt ist. (Ganz zu schweigen von der Frage, inwiefern es sich dabei bereits um Wissenschaft im Sinne der Digital Humanities handelt.) Defizitär erscheint der Ausbildungskanon auf diesem Feld auch deshalb, da allein 16 von 28 Institutionen in den Untersuchungszeiträumen gerade einmal mit einer entsprechenden Lehrveranstaltung vertreten sind, die sich in der Regel entweder dem Thema digitaler Notensatz oder computergestützte Musikanalyse gewidmet hat. Insgesamt führend ist auch hier wieder (mit fünf Lehrveranstaltungen) das musikwissenschaftliche Seminar Detmold/Paderborn, gefolgt von den Instituten an den Universitäten Bremen, Mainz, Köln und Regensburg (mit jeweils vier Lehrveranstaltungen).

Mit Blick auf die Fragestellung nach den digitalen Dimensionen heutigen musikwissenschaftlichen Arbeitens lassen sich aus den vorangegangenen Betrachtungen folgende vier großen Bereiche herausfiltern: 1. Digitale Editionsarbeit (Musik- und Textedition) - 2. Digitale Quellenarbeit (Erschließung, Aufbereitung und Präsentation) 3. Erstellen digitaler Enzyklopädien und Bibliographien, einschließlich der Überführung bereits vorliegender analoger Informationen in digitale Umgebungen - 4. Computergestützte Musikanalyse.

\subsection{Zum Gegenstand der Arbeit}

Nach diesem Blick auf den Status quo digitalen musikwissenschaftlichen Arbeitens kann nun noch einmal die eingangs gestellte Frage nach der digitalen Entwicklungsstufe des Faches Musikwissenschaft aufgegriffen werden. Die eben herausgefilterten vier großen Bereiche betreffen zunächst alle vier ${ }^{49}$ von Gardner und Springfeld beschriebenen Schritte wissenschaftlichen Arbeitens (Literatur- und Quellenrecherche, Materialsichtung,

\footnotetext{
${ }^{49}$ Der erste der fünf Schritte, das Finden und Definieren einer Fragestellung muss hier freilich außen vor bleiben (siehe bei Fußnote 12).
} 
Materialbewertung, Darstellung) und zeigen, dass die Fachcommunity Methoden und Werkzeuge etabliert hat, um im digitalen Umfeld Wissenschaft zu betreiben.

Das alles sagt aber noch nichts darüber aus, erstens, wie verbreitet diese Methoden und Werkzeuge im Fach inzwischen sind, zweitens, ob dies schon als wissenschaftliche Arbeitsweise im Sinne der Digital Humanities verstanden werden darf und drittens, wie mit den im digitalen Forschungsprozess entstehenden Daten - von der Literatur- und Quellenverwaltung, über die Datenverwaltung im Prozess der Erhebung und Bewertung bis schließlich zur Präsentation der Ergebnisse - umgegangen wird.

Alle drei Punkte sind eng miteinander verknüpft. Die Methoden der Digital Humanities sind sehr stark auf eine wenigstens disziplinäre, in der Regel aber sogar interdisziplinäre Vernetzung und Kontextualisierung des Wissen ausgerichtet. Dieses Ziel kann aber nur erreicht werden, wenn in der wissenschaftlichen Arbeit methodische Standards und technische Richtlinien Anwendung finden, die für alle Mitglieder dieser disziplinären oder interdisziplinären Community gelten. Eine Verständigung des Faches über Datenformate, Metadatenschemata und Schnittstellen, aber auch Konzepte zur Speicherung und zum Verfügbarmachen der erhobenen Daten ist unerlässlich, um die Rahmenbedingungen zu schaffen, damit sich eine digital vernetzte Fachdisziplin entwickeln kann.

Ein derartiges Konzept existiert für das Fach Musikwissenschaft bisher nicht. Eine Verständigung darüber hat gerade erst begonnen. Im Herbst 2016 hat sich der Beirat der Gesellschaft für Musikforschung daher an die einzelnen Fachgruppen der Gesellschaft gewandt, um diese zur Bildung einer Arbeitsgemeinschaft mit Vertretern mehrerer Fachgruppen anzuregen. Ziel der Arbeitsgruppe soll es sein, sich mit den Leitlinien zum Umgang mit Forschungsdaten der Deutschen Forschungsgemeinschaft auseinandersetzen und ein fachspezifisches Konzeptpapier zu erarbeiten. ${ }^{50}$ Auf diesen Aufruf hin haben sich Vertreter der Fachgruppen Musikinstrumentenkunde, Frauen- und Genderstudien, Systematische Musikwissenschaft, Musikethnologie und vergleichende Musikwissenschaft und Freie Forschungsinstitute gemeldet und es wurde im Folgenden beschlossen, dass diese Vertreter zunächst fachgruppenspezifische Konzepte erarbeiten, die erst anschließend zusammengefügt und harmonisiert werden. Ich selbst bin an dieser Arbeitsgruppe als ein Vertreter der Freien Forschungsinstitute beteiligt.

\footnotetext{
${ }^{50}$ DFG-Leitlinien zum Umgang mit Forschungsdaten. Gründung einer GfM-Arbeitsgruppe, Email von Christiane Wiesenfeldt an die Fachgruppensprecherinnen und -sprecher der Gesellschaft für Musikforschung vom 28. Oktober 2016.
} 
Bei den sogenannten „freien“ Forschungsinstituten handelt es sich um die „,von den Universitäten finanziell und inhaltlich unabhängigen“ Forschungseinrichtungen. Etwa die Hälfte dieser derzeit 50 Institute arbeitet an „Editionen der großen Musiker-Gesamtausgaben Die andere Hälfte ist in archivalischen, quellenkundlichen oder musealen Bereichen tätig und oft ebenfalls mit Editionen oder Grundlagenforschung befasst. “51

Die Arbeit der Freien Forschungsinstitute fällt damit in das Aufgabenprofil der Historischen Musikwissenschaft. Hinsichtlich der oben beschriebenen digitalen Arbeitsfelder musikwissenschaftlichen Arbeitens sind die wesentlichen Herausforderungen hier die Themen digitale Editionsarbeit und digitale Quellenarbeit.

Mit Blick auf die im Fach akzeptierte und weitgehend strikte Unterscheidung zwischen Historischer und Systematischer Musikwissenschaft ${ }^{52}$ scheint mir die Trennung und Fokussierung auch hinsichtlich der hier diskutierten Arbeitsfelder sinnvoll - einerseits, da beide Teildisziplinen mit sehr verschiedenen Methoden arbeiten und sehr verschiedene Forschungsdaten erzeugen und hier ohnehin getrennt betrachtet werden müssten und zweitens, um den äußeren Rahmen dieser Arbeit nicht zu sprengen.

Im Folgenden soll daher der Forschungsfrage nachgegangen werden, wie das Forschungsdatenmanagement in der musikwissenschaftlichen Editions- und Quellenarbeit organisiert ist und welche Veränderungen sich durch die zunehmend digitale Ausrichtung dieser Wissenschaftsfelder für das Forschungsdatenmanagement ergeben. Es soll geschaut werden, welche Probleme daraus erwachsen und wie diesen Problemen disziplinspezifisch begegnet werden kann.

In einem ersten Schritt gilt es daher zu schauen, welche Forschungsdaten im Umfeld musikwissenschaftlicher Editions- und Quellenarbeit im analogen Zeitalter angefallen sind,

\footnotetext{
${ }^{51}$ Gesellschaft für Musikforschung. Fachgruppe Freie Forschungsinstitute (http://www.musikforschung.de/index.php/fachgruppen/freie-forschungsinstitute).

${ }^{52}$ Die Unterteilung der Musikwissenschaft in eine historische und eine systematische Teildisziplin geht auf Guido Adler zurück, der sie erstmals 1885 formuliert und 1919 in seiner Methode der Musikgeschichte nochmals bekräftigt hat. Unter der Historischen Musikwissenschaft versteht er die „Geschichte der Musik nach Epochen, Völkern, Reichen, Ländern, Gauen, Städten, Schulen, Künstlern“. Zu ihren Teilen zählt er „A. Musikalische Palaeographie (Semeiographie) (Notationen). B. Historische Grundklassen (Gruppierung der musikalischen Formen). C. Gesetze: 1. wie sie in den Kunstwerken je einer Epoche vorliegen, 2. wie sie von den Theoretikern der betreffenden Zeit gefaßt und gelehrt werden, 3. wie sie in der Kunstausübung hervortreten. D. Musikalische Instrumente.“ Aufgabe der Systematischen Musikwissenschaft ist für ihn die „Aufstellung der in den einzelnen Zweigen der Tonkunst zu höchst stehenden Gesetze“ Zu ihren Teilen zählt er die „A. Erforschung und Begründung derselben in: 1. Harmonik (tonal), 2. Rhythmik (zeitlich), 3. Melik (Korrelation von tonal und zeitlich). B. Ästhetik und Psychologie der Tonkunst: 1. Vergleichung und Wertschätzung und deren Relation mit den apperzipierenden Subjekten, 2. Komplex unmittelbar und mittelbar damit zusammenhängender Fragen. C. Musikalische Pädagogik und Didaktik: 1. Allgemeine Musiklehre, 2. Harmonielehre, 3. Kontrapunkt, 4. Kompositionslehre, 5. Instrumentationslehre, 6. Methoden des Unterrichtes in Gesang und Instrumentalspiel. D. Musikologie (Untersuchung und Vergleichung im Dienste der Ethnographik und Folkloristik).“ Siehe Adler 1919, S. 7.
} 
mit Blick auf welche Bedürfnisse diese hier verwaltet wurden und wie sie heute zur Verfügung stehen. Danach soll durch die exemplarische Betrachtung neuerer Forschungsprojekte geschaut werden, wie sich der Digital Turn in der Musikwissenschaft aktuell vollzieht und welche Auswirkungen dies auf den Umgang mit den Forschungsdaten hat. Abschließend sollen die musikwissenschaftlichen Bedürfnisse zum Umgang mit Forschungsdaten mit den Leitlinien zum Umgang mit Forschungsdaten der Deutschen Forschungsgemeinschaft und den weitgehend identischen Grundsätzen zum Umgang mit Forschungsdaten der Allianz der deutschen Wissenschaftsorganisationen abgeglichen und Empfehlungen für ein fachspezifisches Konzept zum Forschungsdatenmanagement für die musikwissenschaftlichen Editions- und Quellenarbeit formuliert werden.

\section{Forschungsdaten in der musikwissenschaftlichen Editions- und Quellenarbeit}

Die Musikwissenschaft wird oft als eine der jüngsten Wissenschaftsdisziplinen beschrieben, und tatsächlich existiert sie als Universitätsfach erst seit gut einem Jahrhundert. ${ }^{53}$ Hugo Riemann, der zusammen mit Guido Adler als Vater der Musikwissenschaft gilt, wurde 1901 an der Universität Leipzig zum außerordentlichen Professor ernannt und begründete 1908 in Leipzig das erste musikwissenschaftliche Institut, dessen Direktor er wurde. Die theoretische Beschäftigung mit Musik ist freilich schon viel älter und reicht zurück bis in die Antike. Fragen der Musikgeschichtsschreibung erhielten seit dem 18. Jahrhundert einen akademischen Skopus, wofür sich seit dieser Zeit Begriffe wie „musikalische Wissenschaft“, „Tonwissenschaft““, „Musikforschung“ oder „Musikologie“ entwickelten.

\section{1. ,Analoges Forschungsdatenmanagement' in der Musikedition und der Arbeit mit musikalischen Quellen am Beispiel der Bach-Gesamtausgabe}

Die wissenschaftliche Musikedition und Musikphilologie ist ein Phänomen, das im 19. Jahrhundert entstand, gleichwohl das Bewusstsein um Werktreue und Originalität schon bis

\footnotetext{
${ }^{53}$ Dies und das Folgende nach den Artikeln Musikwissenschaft, in: MGG, Bd. 9, Sp. 1192ff. und MGG², Sachteil, Bd. 7, Sp. 1789ff. sowie Musicology, in: GMO - zuletzt aufgerufen am 24. April 2017.
} 
ins 18. Jahrhundert zurückreicht. ${ }^{54}$ Die erste wissenschaftliche Musiker-Gesamtausgabe wurde 1850 mit der Gesamtausgabe Johann Sebastian Bach's Werke, herausgegeben von der zu diesem Zweck gegründeten Bach-Gesellschaft und erschienen beim Verlagshaus Breitkopf \& Härtel, begonnen. Ihre Prinzipien folgen in vielerlei Hinsicht bereits den Ansprüchen heutiger Ausgaben „und müssen daher als ,modern“ bezeichnet werden“. 55

Die ersten beiden Bände mit Kirchenkantaten wurden von Moritz Hauptmann vorgelegt. Er gehörte zu den Gründungsmitgliedern der Bach-Gesellschaft, war von 1842 bis zu seinem Tod im Jahr 1868 Thomaskantor in Leipzig und außerdem Lehrer am Leipziger Konservatorium. ${ }^{56}$ Spätere hauptverantwortliche Herausgeber waren Wilhelm Rust, Thomaskantor von 1880 bis 1892 und verantwortlich für 26 Bände der Ausgabe, ${ }^{57}$ sowie Alfred Dörffel, Musikbibliothekar in Leipzig und Redakteur, unter anderem für die Neue Zeitschrift für Musik. ${ }^{58}$ Nach knapp 50 Jahren Bearbeitungszeit konnte die Ausgabe in 46 Jahrgangsbänden (und 60 Teilbänden) 1899 abgeschlossen und die Bach-Gesellschaft im darauffolgenden Jahr aufgelöst werden.

Die Bände dokumentieren in den Kritischen Berichten die wissenschaftliche Arbeitsweise der Herausgeber weitgehend transparent, bieten in den späteren Jahrgängen durchweg Abbildungen von Wasserzeichen und Faksimiles aus den Quellen ${ }^{59}$ und haben mit ihrer Dokumentationsweise Maßstäbe in der editorischen Arbeit gesetzt, die noch weit bis ins 20. Jahrhundert hinein gewirkt haben.

Nicht publizierte Unterlagen - oder modern gesprochen Forschungsdaten -, die in so einem klassischen Editionsprozess anfallen, sind:

\footnotetext{
${ }^{54}$ Noch bis ins frühe 19. Jahrhundert fand die Distribution von musikalischen Werken hauptsächlich und in der Masse über handschriftliche Kopienahme statt. (Zu dem Phänomen siehe etwa Glöckner 1984, Reipsch 2001, Maul/Wollny 2003 sowie Kepper 2011, S. 20.) Erst neue technische Entwicklungen im Notendruck, die sich seit der Mitte des 18. Jahrhunderts Bahn brachen, führten zu einer immer stärkeren Zunahme des Drucks musikalischer Werke und waren die Voraussetzung für die wissenschaftliche Editionsarbeit. Der Musikalienverleger Johann Gottlob Immanuel Breitkopf hatte in den 1750er Jahren in Leipzig damit begonnen auf Grundlage einer neuentwickelten Satztechnik überregional beachtete Musikdrucke herzustellen und verfolgte gemeinsam mit dem Dresdner Hofkapellmeister Johann Adolf Hasse zu dieser Zeit das Ziel eine Gesamtausgabe von dessen Opern vorzulegen. In einem Subskriptionsaufruf vom November 1756 schreibt Breitkopf: „Die Liebhaber, welche bisher die Haßischen Werke verlangten, waren genöthiget, sich an Notenschreiber zu wenden, worunter viele aus Unwissenheit oder aus Nachläßigkeit öffters so falsch schrieben, daß der Compositeur kaum selbsten seine Arbeit kannte.“ Die Wirren des Siebenjährigen Krieges (1756-1763), der für ganz Sachsen schwere wirtschaftliche Folgen hatte, verhinderten das Zustandekommen der Ausgabe schließlich allerdings. (Bärwald 2016, Bd. 1, S. 74ff.).

${ }^{55}$ Kepper 2011, S. 27.

${ }^{56}$ Hauptmann, Moritz, in: MGG, Bd. 5, Sp. 1828ff.

${ }^{57}$ Rust, Wilhelm, in: MGG, Bd. 11, Sp.1193ff. sowie Kepper 2011, S. 39ff.

${ }^{58}$ Dörffel, Alfred, in: MGG, Bd. 3, Sp. 621f. Weitere Herausgeber waren Carl Ferdinand Becker, Julius Rietz, Franz Kroll, Franz Espagne, Paul Graf von Waldersee, Ernst Naumann, Franz Wüllner und Hermann Kretzschmar (siehe Kepper 2011, S. 285ff.).

${ }^{59}$ Kepper 2011, S. 285ff.
} 
- Spartierungen von Stimmmaterialien und Partiturabschriften

- Kollationsbögen, in denen die Lesarten in den verschiedenen Quellen und Quellenschichten graphisch zusammengeführt werden

- Dokumentationen von Schreiberhänden und Wasserzeichen sowie Aufzeichnungen, die im Rahmen der Quellenbewertung entstehen

- Stichvorlagen der Notenbände

- Manuskripte der Kritischen Berichte

- Korrekturfahnen, die im Rahmen des Druckprozesses entstehen

- Korrespondenzen zwischen dem Bearbeiter des einzelnen Bandes, den Herausgebern der Gesamtausgabe und dem Verlag

Im Fall der Bach-Gesamtausgabe des 19. Jahrhunderts gelangten solche Unterlagen jahrgangsweise in das Verlagsarchiv von Breitkopf \& Härtel, wo große Teile der Bestände bei der Bombardierung Leipzigs im Zweiten Weltkrieg vernichtet wurden. ${ }^{60} 1962$ wurden die erhaltenen Restbestände an das Staatsarchiv Leipzig übergeben und zunächst über eine Findkartei erschlossen. 1990 wurde diese Kartei in ein Findbuch überführt, wobei der immer noch sehr umfangreiche Teilbestand zur Bach- Gesamtausgabe fälschlicherweise lediglich unter einer einzigen Jahrgangsnummer verzeichnet wurde. Erst im Zuge „einer 2011 erfolgten Neuregelung des Depositalvertrags zwischen Archiv und Verlag wurde 2012 [...] eine große Zahl an Fehlnummern festgestellt; aus diesem Grund wurde dieser Teilbestand noch einmal systematisch erfaßt. Dabei blieb die ursprüngliche jahrgangsweise einzelnen Bänden der $\mathrm{B}$ [ach-]G[esamtausgabe] zugeordnete Aufteilung weitgehend erhalten. “61 Die bis zu diesem Zeitpunkt vollständig in Vergessenheit geratenen Unterlagen stehen der Forschung nun wieder zur Verfügung und wurden bereits einer ersten Auswertung durch Mitarbeiter des Bach-Archivs unterzogen. Hierbei konnten zahlreiche Zimelien ans Licht gebracht werden, darunter wertvolle Musikhandschriften des 18. Jahrhunderts, die im Zusammenhang mit der Entstehung der Bach-Gesamtausgabe im 19. Jahrhundert in diesem Teilbestand des Verlagsarchivs abgelegt wurden. ${ }^{62}$ Für die Entstehung der Neuen Bach-Ausgabe, ${ }^{63}$ die zwischen 1954 und 2007 vom Johann-Sebastian-Bach-Institut in Göttingen und dem Bach-

\footnotetext{
${ }^{60}$ Dies und das Folgende nach Blanken 2013, S. 79 und 84.

${ }^{61}$ Ebenda, S. 80.

${ }^{62}$ Ebenda, S. 80f. und 117-128.

${ }^{63}$ Johann Sebastian Bach. Neue Ausgabe sämtlicher Werke, hrsg. vom Johann-Sebastian-Bach-Institut Göttingen und vom Bach-Archiv Leipzig, Kassel 1954-2007.
} 
Archiv Leipzig bei Bärenreiter herausgegeben wurde, konnten die zu dieser Zeit unbekannten Unterlagen nicht herangezogen werden.

Das Beispiel verdeutlicht sehr schön die Probleme, denen die Verwaltung von

Forschungsdaten in der analogen Welt begegnen kann. Nachdem die Unterlagen nach einem ordnungsgemäßen Verfahren in das Verlagsarchiv überführt und dort sicher auch entsprechend verzeichnet wurden, hat sich für Jahrzehnte niemand für die Materialien interessiert; die wissenschaftliche Arbeit war abgeschlossen und alle für die Zeitgenossen wichtigen Informationen lagen in der gedruckten Gesamtausgabe vor. Als ein Jahrhundert nach Beginn der ersten Gesamtausgabe die Arbeit an der Neuen Bach-Ausgabe aufgenommen wurde, geschah dies vor allem mit Blick auf die Mängel in der Dokumentation der der alten Ausgabe zugrunde liegenden Quellen. ${ }^{64}$

„So verständlich die Hochschätzung der alten Gesamtausgabe und die daraus folgende Kritiklosigkeit ihr gegenüber auch gewesen sein mag, so folgenschwer war sie angesichts der Zerstörung im Zweiten Weltkrieg, denen zahlreiche Quellen zum Opfer gefallen sind. Hätte man die inzwischen verfügbar gewordenen Mittel der modernen Technik bereits vor 1945 zur Dokumentation und zur Handschriftenuntersuchung eingesetzt, so wären die Kriegsverluste leichter zu verschmerzen gewesen. “65

Mit Blick auf die Materialien zur alten Gesamtausgabe, die sich in Verlagsbesitz befanden, und während des Krieges teilweise zerstört wurden, ist der Verlust in diesem Fall sogar ein zweifacher gewesen - und ein dreifacher, wenn man bedenkt, dass die erhaltenen Unterlagen derart in Vergessenheit geraten waren, dass sie für die Neuausgabe nicht herangezogen wurden.

So kommt es bei der Verwaltung von Forschungsmaterialien nicht nur darauf an, für deren physische Unversehrtheit zu sorgen, sondern es ist genauso wichtig, sie auf eine Art und Weise zu dokumentieren, die eine Wiederauffindung erstens auch nach sehr langer Zeit ermöglicht und zweitens selbst dann zielführend ist, wenn bereits das Vorhandensein der Materialien unbekannt oder zumindest aus dem kollektiven Gedächtnis verschwunden ist. Der erste Punkt ist relativ leicht durch eine regelmäßige Inventarisierung der Bestände und eine ständige Aktualisierung der Findmittel zu erreichen. Der zweite Punkt ist hingegen in einer

\footnotetext{
${ }^{64}$ Kepper 2011, S. 89 und 91.

${ }^{65}$ Musikalisches Erbe und Gegenwart. Musiker-Gesamtausgaben in der Bundesrepublik Deutschland, hrsg. von Hanspeter Bennwitz, Kassel 1975, S. 18f., zitiert nach Kepper 2011, S. 91.
} 
rein analog arbeitenden Wissenschaft kaum sinnvoll realisierbar. Hier eröffnen erst die Möglichkeiten der digitalen Katalogisierung und der weiteren Erfassung dieser Daten durch übergeordnete Metakataloge die Chance, Daten- und Materialbestände so zu kontextualisieren, dass sie der forschenden Zielgruppe zumindest unter bestimmten - im Idealfall möglichst breit gefassten - Prämissen zugängig gemacht werden können. Wenn aber schon bei eigentlich so zentral und naheliegenden Beständen, wie dem eben beschriebenen Verlagsarchiv, die Auffindung der Forschungsunterlagen in der analogen Welt beinahe nur per Zufall möglich ist, dann lässt sich erahnen, wie schwierig es ist, noch wesentlich kleinere und verstreutere Bestände zu ermitteln.

Nehmen wir das Beispiel von Wilhelm Rust, der 26 Bände der alten Bach-Gesamtausgabe ediert hat. In seinem persönlichen Besitz befanden sich freilich auch Materialien, die direkt und indirekt mit seiner Herausgebertätigkeit in Verbindung stehen. Seien es Musikalien, wie Quellenkopien oder Briefe, in denen er sich über seine Arbeit äußert. Ohne ein Rechercheportal wie Kalliope, das interdisziplinär organisiert ist und sich sowohl an Bibliotheken, Museen als auch Archive richtet, wäre es nahezu unmöglich mit auch nur annähernd angemessenem Aufwand einen Überblick über das vorhandene Quellenmaterial oder zumindest einen repräsentativen Teil davon, immerhin sind nicht alle denkbaren Bestände in Kalliope verzeichnet - zu verschaffen.

So besitzt das Stadtgeschichtliche Museum Leipzig einen reichen Bestand an Briefen und weiteren Handschriften von und an Wilhelm Rust, von denen einige im Zusammenhang mit der Bach-Gesamtausgabe stehen: mehrere Briefe zwischen ihm und den Mitherausgebern Julius Rietz und Moritz Hauptmann, aber auch Abschriften von Bach-Werken: So verwahrt das Museum neben zwei Eigenkompositionen ${ }^{66}$ von ihm unter der Signatur A/4652/2009 beispielsweise auch eine autographe Abschrift Rusts von Bachs Rezitativ „Verfolgt dich gleich die arge Welt" aus der Kantate Ach Gott, wie manches Herzeleid BWV 58. Das Werk hatte Rust 1863 innerhalb eines Bandes mit zehn Kirchenkantaten herausgegeben. ${ }^{67}$ Eine weitere Bestandsgruppe - es handelt sich um einen Teil des Nachlasses von Rust besitzt das Bach-Archiv Leipzig. Auch dieser ist bei Kalliope verzeichnet, ${ }^{68}$ während andere Rust-Manuskripte, die dieselbe Institution besitzt, hier nicht auftauchen. Das wiederum hängt mit Gründen der Provenienz zusammen. In dem Teilbestand Sammlung Manfred Gorke

\footnotetext{
${ }^{66}$ Stadtgeschichtliches Museum Leipzig, A/5244/2009 und A/5246/2009.

${ }^{67}$ Johann Sebastian Bach, Kirchencantaten. Band 6, hrsg. von Wilhelm Rust, Leipzig 1863 (Johann Sebastian Bach's Werke. 12,2.).

${ }^{68}$ Kalliope DE-611-BF-13503 (http://kalliope-verbund.info/DE-611-BF-13503).
} 
bewahrt das Bach-Archiv mehrere Notenautographe Rusts auf; auch hierbei handelt es sich größtenteils um Abschriften Bachscher Werke. ${ }^{69}$ Außerdem umfasst der Bestand mehrere Konvolute mit Briefen und Postkarten an Rust - unter anderem von Moritz Hauptmann und dem Bach-Biographen Philipp Spitta. Da die Sammlung Manfred Gorke im Bach-Archiv stets als Musikaliensammlung behandelt wurde, sind bisher auch nur deren Musikalienbestände über das RISM bibliographisch erfasst worden. Erst ein im Herbst 2016 gestartetes Digitalisierungs- und Erschließungsprojekt, das den gesamten Bestand der Sammlung erfassen soll, wird hier Abhilfe schaffen, indem sämtliche Objekte der Sammlung über den Verbundkatalog des Südwestdeutschen Bibliotheksverbundes recherchierbar sein werden. ${ }^{70}$

Das Beispiel der alten Bach-Gesamtausgabe und der Arbeitsunterlagen und wissenschaftlichen Korrespondenz von Wilhelm Rust machen deutlich, dass ein einheitliches und systematisches Forschungsdatenmanagement in der analogen Welt kaum möglich ist und über einen großen Zeitraum angefallene Materialien nur schwer über Standards harmonisierbar und zentral speicherbar sind, so dass eine Nachnutzung nie ohne Reibungsverluste möglich ist. Die Probleme lassen sich freilich bei einer stärker institutionalisierten und systematischen Vorgehensweise minimieren. Die Neue BachAusgabe entstand an zwei Forschungsinstituten, die Bibliotheks- und Archivstrukturen aufgebaut haben, um vor allem eine langfristige Sicherung von Quellenkopien, Spartierungsund Kollationsbögen, wissenschaftlichen Aufzeichnungen, Stichvorlagen, Manuskripten, Korrekturfahnen und Korrespondenzen zu gewährleisten. Doch auch hier besteht das Problem, dass lange Zeit nicht erkannt wurde, dass diese Materialien nur nach einer systematischen Erschließung auch tatsächlich zugängig und nachnutzbar sind. ${ }^{71}$ Für die gesammelten Kopien originaler Musikalienquellen, die aus Bibliotheken und Archiven weltweit zusammengetragen wurden, wurde am Bach-Archiv eine Findkartei geführt, die das ermöglicht hat. Eine Erschließung der übrigen Materialien ist hingegen nicht erfolgt. Als das Johann-Sebastian-Bach-Institut Göttingen Ende 2006 geschlossen wurde, gelangten dessen Archivmaterialien ins Bach-Archiv nach Leipzig, und es wurde in der Folge damit begonnen die über Jahrzehnte gesammelten Quellenkopien sowohl über den Onlinekatalog der

\footnotetext{
${ }^{69}$ Schulze 1977, Nr. 11, 29, 30, 31, 142, 143, 267, 269, 271, 273, 274, 276, 278, 280, 283, 284, 286-288, 319 und 320 .

${ }^{70}$ Siehe die ausführlichere Darstellung des Projektes in Abschnitt 3.3.2. dieser Arbeit.

${ }^{71}$ Bei Diesem und dem Folgendem handelt es sich um einen Erfahrungsbericht des Autors dieser Arbeit, der als wissenschaftlicher Mitarbeiter im Forschungsinstitut und wissenschaftlicher Referent der Forschungsbibliothek des Bach-Archivs dort unter anderem für die Einführung von Strukturen und elektronischen Systemen für die Forschungsdaten- und Dokumentationsdatenverwaltung zuständig ist.
} 
Bibliothek des Bach-Archivs als auch innerhalb der Quellendatenbank der Forschungsplattform Bach digital zu verzeichnen. Heute ist dieser Materialkorpus hier vollständig erschlossen und frei recherchierbar.

Mit den übrigen, bisher nicht aufgearbeiteten Materialien soll in 2017 begonnen werden, ein wissenschaftliches Hausarchiv aufzubauen als dessen Grundlage derzeit ein Akten- und Registraturplan erarbeitet wird, der zukünftig auch für die laufenden wissenschaftlichen Projekte und Verwaltungsstrukturen Gültigkeit besitzen soll. Außerdem wird dieser Aktenplan zugleich die strukturelle Grundlage für ein ebenfalls in $2017 \mathrm{zu}$ etablierendes elektronisches Dokumenten- und Medienmanagementsystem sein, das als digitale Forschungs- und Dokumentationsumgebung mit Webschnittstelle zugleich als Tor für die digitale Nachnutzung der elektronisch verwalteten Forschungs- und Dokumentationsdaten dienen kann.

\subsection{Musikeditorisches und quellengebundenes Arbeiten als ,Small Science‘?}

Trotz des Einsatzes von elektronischen Systemen zur Verwaltung dieser Daten wird es aufgrund der höchst individualisierten Arbeitsweisen der Forschenden, die nur selten in größeren Teams organisiert sind, auch in Zukunft schwer sein, ein einheitliches, ganzheitliches und systemisches Forschungsdatenmanagement zu etablieren. Das liegt auch daran, dass die musikwissenschaftliche Editions- und Quellenarbeit keine datengetriebene Wissenschaft ist, und damit die Notwendigkeit von Nutzen und Umfang einheitlicher Standards und elektronischer Systeme zur Forschungsdatenverwaltung sehr unterschiedlich interpretiert wird - sofern eine Auseinandersetzung mit dem Thema überhaupt stattfindet. Einschlägige Untersuchungen, auch für die Musikwissenschaft und ihre Teilbereiche fehlen bisher.

Die von Jim Grey als viertes Forschungsparadigma beschriebene datengetriebene Wissenschaft (Data-Intensive Scientific Discovery) $)^{72}$ eröffne durch die „,informationstechnologische Verknüpfung der heterogenen Datenmengen [...] neue Dimensionen wissenschaftlicher Erkenntnis“.${ }^{73}$ Die Redeweise vom vierten Paradigma bezieht sich auf die Wissenschaftsparadigmen Empirie und Theorie, denen sich historisch durch die Entwicklung neuer informationstechnologischer Lösungen die Möglichkeit der

\footnotetext{
${ }^{72}$ Gray 2009.

${ }^{73}$ Müller 2011, S. 131.
} 
Darstellung komplexer Phänomene in Simulationen anschließt. ${ }^{74}$ In der datengetriebene Wissenschaft entwickeln sich inzwischen ,gänzlich neue Wege zu wissenschaftlicher Erkenntnis auf der Grundlage der Exploration von massenhaft vorhandenen oder erhobenen Daten“. 75

Lars Müller hat die Arbeit der datengetriebenen Wissenschaft am Beispiel eines so datenintensiven Bereichs wie der Klimaforschung verdeutlicht und gezeigt, wie das Forschungsdatenmanagement einer Klimaplattform organisiert sein muss, indem es der Entwicklung einer Forschungsdateninfrastruktur bedarf, die „die Übergänge zwischen Disziplinen, Small und Big Science, Institutionen sowie technischen Systemen schafft.“ Voraussetzungen hierfür seien „die Einführung von Forschungsdatenpolicies, Semantic Web Technologien und Servicestrukturen für das Forschungsdatenmanagement“ ${ }^{76}$

In der musikwissenschaftlichen Forschung, in der Erarbeitung von wissenschaftlichen Editionen und der Erschließung und Aufbereitung musikalischer Quellen, entstehen durch die Digitalisierung immer größere Datenmengen. Dabei handelt es sich vor allem um große Bestände von Quellendigitalisaten, die in Datenbanken und Repositorien wie Bach digital oder den Digitalen Sammlungen der SLUB Dresden vorgehalten, mit oft umfangreichen Metadatenumgebungen versehen und mit externen Datenbeständen verknüpft werden, wie das beispielsweise im RISM geschieht. Sind diese Strukturen aber schon hinreichend, um hier von einer datengetriebenen Wissenschaft im Sinne einer Big Science zu sprechen? Dies würde beispielsweise das Vorhandensein fachdisziplinspezifischer Dateninfrastrukturen voraussetzen, also spezifische Datenzentren, in denen diese Daten erfasst und verwaltet werden. ${ }^{77}$ Das ist für die Musikwissenschaft nicht der Fall, und das Fach ist auch nicht auf dem Weg dahin. Vielmehr sind es - im Sinne einer Small Science - „überschaubare Forschungsprojekte“, die sich mit „spezifischen Fragen“ beschäftigen und von „wenigen Einzelpersonen durchgeführt" werden: ${ }^{78}$

„Die anfallenden Daten befinden sich dann am Arbeitsplatz der Wissenschaftler, einen Austausch gibt es innerhalb der Arbeitsgruppe, darüber hinaus findet nur wenig Austausch statt. Nach Projektabschluss ist der Verbleib der Daten ungewiss, oft gehen sie verloren. Policies und Datenmanagementpläne existieren nicht, sind nicht bekannt

\footnotetext{
${ }^{74}$ Büttner 2011, S. 14.

${ }^{75}$ Ebenda.

${ }^{76}$ Müller 2011, S. 131.

${ }^{77}$ Siehe die Ausführungen zum Thema „Data Center“ ebenda, S. 134.

${ }^{78}$ Dies und das Folgende ebenda, S. 133.
} 
oder werden nicht eingehalten. Selbstverständlich findet in den Arbeitsgruppen ein spezifisches Datenmanagement statt. Allerdings dominieren projektbezogene Lösungen, wie die Ablage von Daten in einem einfachen Dateisystem. Faktisch sind bei ,selbstgestrickten“ Lösungen die Daten ohne das Wissen der beteiligten Forscher wertlos. Selbst wenn zentrale Services wie ein Datenrepository in der Institution existieren, werden sie nicht zwangsläufig genutzt. Als besonders problematisch erweist sich die sorgfältige Erschließung mit Metadaten, die für die Nachnutzung von Forschungsdaten unabdingbar, aber in der Praxis nur schwer umzusetzen ist, denn sie ist mühsam und zeitintensiv.“

Auf die nicht vorrangig digital arbeitenden musikwissenschaftlichen Forschungsprojekte lässt sich diese Feststellung sicher weitgehend übertragen. Wie sieht es aber bei jenen Forschungsprojekten aus, die sich das digitale Arbeiten auf die Fahne geschrieben haben und unter dem Anspruch arbeiten, Methoden und Werkzeuge der Digital Humanities zu nutzen, weiterzuentwickeln oder zu etablieren? Oder anders gefragt, von welchen Einflüssen und Entwicklungen, ist der Digital Turn in der quellenbasiert arbeitenden Musikwissenschaft geprägt?

\section{Der ,Digital Turn` in der Musikwissenschaft und seine Konsequenzen für den Umgang mit fachspezifischen Forschungsdaten in den Bereichen Musikedition und musikalische Quellenarbeit}

Digitales Arbeiten erfasst inzwischen alle Bereiche der Wissenschaft, indem fachspezifische Online-Nachschlagewerke und Datenbanken entstehen, Online-Journale und eBooks herausgegeben werden und analoge Medien in digitale Umgebungen überführt werden. Bibliotheken digitalisieren ihre Altbestände, bieten Virtuelle Fachbibliotheken an und ermöglichen im Rahmen der von der Deutschen Forschungsgemeinschaft geförderten Fachinformationsdienste viele weitere Online-Services für ihre Nutzer.

Eine allgemeingültige Definition des Digital Turn, unter dem landläufig die Transformation der Informations- und Wissensgesellschaft vom Analogen ins Digitale verstanden wird, gibt 
es nicht. ${ }^{79}$ Entsprechend schwierig ist es auch, die Veränderungen, die sich durch den digitalen Wandel in den Wissenschaften einstellen, qualitativ zu bewerten und zu verstehen, was ihre Auswirkung auf die Wissensproduktion und die Informationsservices - kurz- und langfristig, für den einzelnen Wissenschaftler wie auch für die gesamte Disziplin, institutionsintern aber erst recht global - bedeuten.

\subsection{Digitale Musikwissenschaft}

Sind Wissenschaften, die vom digitalen Wandel ergriffen werden, zunehmend elektronisch arbeiten und digitale Werkzeuge verwenden, automatisch digitale Wissenschaften? Es würde diese Behauptung bedeuten, dass eine „Musikwissenschaft“ zugunsten einer „Digitalen Musikwissenschaft“ nach und nach verschwindet. Dieser ganz unwissenschaftliche Gedankengang kann nicht überzeugen. Vielmehr sollte der Begriff der Digitalen Musikwissenschaft im Sinne einer eigenständigen Disziplin oder vielmehr Teildisziplin verstanden werden, die nicht einfach nur die klassischen Aufgabenfelder musikwissenschaftlichen Arbeitens in digitale Arbeitsumgebungen überführt, sondern zu einer neuen interdisziplinär ausgerichteten Forschungsmethodik gelangt, indem beispielsweise gezielt musikwissenschaftliche Semantic Web-Inhalte und fachspezifische Ontologien aufgebaut werden. Insofern scheint mir Wolfgang Schmales Ansatz zur Bestimmung einer „Digital Musicology im Kontext der Digital Humanities“ kritikwürdig: „Dem Augenschein nach ist die Frage, ob sich die Musikwissenschaft auf dem Weg zur Digitalen Musikwissenschaft befindet, klar mit ja zu beantworten. “80 Dies scheint darauf abzuzielen, dass es bei den Digital Humanities darum geht, die vor-digitale Wissenschaft zu überwinden und sie in eine digitale zu transformieren, indem die klassischen Methoden wissenschaftlichen Arbeitens durch digitale Werkzeuge und Arbeitsumgebungen ersetzt werden. Dies wird auch in dem folgenden Statement deutlich: „Sich in einer Geistes- und Kulturwissenschaft zu betätigen, heißt derzeit und noch lange, über eine Doppelkompetenz verfügen zu müssen. Man muss die jeweilige Wissenschaft, wie sie historisch gewachsen ist, verstehen und sich in ihr bewegen können - simpel ausgedrückt, man muss z. B. ,analoge Recherchemethoden kennen und praktizieren können - und man muss dieselbe Wissenschaft als digitale Wissenschaft kennen und handhaben.“ Einschränkend fügt er schließlich allerdings hinzu: „Ob es sich bei der digitalen tatsächlich um ,dieselbe Wissenschaft‘ handelt,

\footnotetext{
${ }^{79}$ Vergleiche dazu auch die Überlegungen bei Kossek 2012.

${ }^{80}$ Schmale 2016, S. 299.
} 
ist eine offene Frage. Die Antwort besteht nicht in einem schlichten ja. ${ }^{681}$ Ich sehe nicht, dass sich die Dinge so simpel auseinander dividieren lassen und es nur Zeit braucht, bis die analoge Wissenschaft in eine vollständig digitale überführt ist. Die Digital Humanities betreiben nicht einfach dieselbe Wissenschaft mit digitalen Mitteln, sondern stellen sich ganz neuen Fragen und Aufgaben, während auch die klassischen Forschungsfelder weiterbestehen, ${ }^{82}$ aber natürlich zunehmend mit digitalen Werkzeugen arbeiten werden, ohne dass man in diesem Fall erstens von einer digitalen Wissenschaft sprechen sollte, sondern vielmehr von digitalem wissenschaftlichem Arbeiten oder von wissenschaftlichem Arbeiten unter Verwendung digitaler Werkzeuge und Arbeitsumgebungen. Zweitens kann es nicht das Ziel dieses Prozesses sein, über Jahrzehnte - zum Teil Jahrhunderte - erprobte und entwickelte Methoden des wissenschaftlichen Arbeitens dem kollektiven Vergessen anheimzustellen. Wenn einmal alle historischen Quellentexte digitalisiert und in eine maschinenlesbare Sprache übersetzt sein sollten, braucht es dann nicht immer noch Wissenschaftler, die in der Lage sind, die beispielsweise in deutscher Kurrentschrift verfassten Originalmanuskripte sicher zu lesen? Schon heute wird diese Fähigkeit im Musikwissenschaftsstudium nicht mehr flächendeckend vermittelt und ist bei Absolventen des Faches eher die Ausnahme als die Regel. Ähnliche Tendenzen zeichnen sich im Umgang mit historischen Notationsweisen ab. Auch vor diesem Hintergrund ist die Etablierung einer Teildisziplin oder Metawissenschaft unter dem Label „Digitale Musikwissenschaft“ innerhalb des Faches dringend notwendig, damit unter den Kapazitäten, die der Digital Turn in der Wissenschaft bindet, das fachspezifische praktische Wissen nicht leidet oder gar in Vergessenheit gerät. ${ }^{83}$ Das Label „Digitale Musikwissenschaft“ darf eben gerade nicht als Modernitätsausweis und omnipräsente Pseudorechtfertigung für Alles und Jedes herhalten müssen: Nicht jede mit einem Notensatzprogramm am Computer erstellte wissenschaftliche Werkausgabe ist eine Forschungsleistung im Sinne der Digital Humanities; auch die Bereitstellung eines Quellendigitalisats oder eines Online-Journals gehört meines Erachtens nicht hierher, solange dabei keine Ansätze verfolgt werden, die zu einer Wissensproduktion

\footnotetext{
${ }^{81}$ Ebenda, S. 302f.

${ }^{82}$ Siehe etwa Wolfgang Auhagens Überzeugung, dass es ,immer Bereiche musikwissenschaftlicher Forschung geben [wird], auf die sich neue Technologien kaum auswirken“ (Müller 2015, S. 11).

${ }^{83}$ Siehe hierzu auch die kritische Positionsbestimmung von Reiner Nägele, der die ,digitale historische Musikwissenschaft“ als „eine fragwürdige Disziplin“ bezeichnet und dabei sogar so weit geht zu vermuten, dass die Musikwissenschaft als eine hermeneutische Disziplin ,,mit den Konzepten und Verfahren und den damit verbundenen Erkenntniswegen der Digital Humanities möglicherweise nicht kompatibel“ (siehe Nägele 2016) ist.
} 
beitragen, die über das jeweilige digitale Objekt - in den interdisziplinären Forschungsraum hinausreicht.

Haben wir oben ${ }^{84}$ von der Musikwissenschaft - oder zumindest der hier betrachteten Teilbereiche Musikedition und quellengebundenes Arbeiten - als einer Small Science gesprochen, so sind die Digital Humanities die Big Science. Die Aufgabe einer Digitalen Musikwissenschaft sehe ich darin, zwischen der Musikwissenschaft und den Digital Humanities als Vermittler zu wirken, mit eigenen exemplarischen Forschungsvorhaben die infrastrukturellen und technischen Möglichkeiten des Faches auszuloten und zu schauen, wie die so gewonnenen Daten für den interdisziplinären Diskurs innerhalb der Digital Humanities im Sinne einer e-Science ${ }^{85}$ bereitgestellte werden können. Mit der Etablierung des Zen $|\mathrm{M}| \mathrm{E} \mid \mathrm{M}$ einerseits und einer Professur für Digitale Musikedition und Digital Humanities andererseits, wie sie in der jüngsten Vergangenheit am Musikwissenschaftlichen Seminar Detmold/Paderborn stattgefunden hat, ${ }^{86}$ scheint sich die deutsche Musikwissenschaft auf einem guten Weg in diese Richtung zu befinden.

\subsection{Digitale Editionsarbeit in der Musikwissenschaft}

Erste Schritte in der Entwicklung eines computerbasierten Notensatzes wurden bereits Ende der 1960er Jahre vollzogen, während der konsequente Wandel hin zu einem hauptsächlich computerbasierten Notensatz erst relativ spät stattfand, indem Bärenreiter erst nach der Wiedervereinigung in den 1990er Jahren gezwungen war, diesen Schritt zu gehen und Henle sogar erst im Jahr 2000 folgte.$^{87}$ Erste Ideen zu Formen digitaler Editionen kamen etwa zur selben Zeit aus der Wissenschaft. Uwe Wolf, seinerzeit wissenschaftlicher Mitarbeiter am Johann-Sebastian-Bach-Institut in Göttingen, beschrieb 2001 seine Idealvorstellung einer EDV-basierten Musikedition, in der man die Möglichkeit habe, vom Notentext mit einem Mausklick zur entsprechenden Stelle im Kritischen Bericht, von dort zur Quellenbeschreibung, zum Faksimile, weiter zur Biographie des Schreibers oder zur Geschichte der Papiermühle und so weiter zu gelangen. ${ }^{88}$ Konkrete Wege in diese Richtung

\footnotetext{
${ }^{84}$ Kapitel 2.2.

85 ,e-Science is about global collaboration in key areas of science, and the next generation of infrastructure that will enable it.“ John Taylor, zitiert nach Büttner 2011, S. 14.

${ }^{86}$ Siehe dazu Kapitel 1.1. dieser Arbeit.

${ }^{87}$ Kepper 2011, S. $127 \mathrm{f}$.

${ }^{88}$ Uwe Wolf, Eine zukünftige ,elektronische NBA' als Arbeitsmittel werkchronologischer Forschung, in: Die Zeit, die Tag und Jahre macht, hrsg. von Martin Staehelin, Göttingen 2001, S. 40f.; hier nach Kepper 2011, S. $130 f$.
} 
beschritt seit 2001 Joachim Veit, indem er nicht nur die Frage nach „,denkbaren Alternativen [...] zur heutigen Editionsform“" stellte, sondern diese durch die Begründung einer engen Zusammenarbeit mit Informatikern als Editionsleiter der Carl-Maria-von-WeberGesamtausgabe und Leiter des Edirom-Projekts entscheidend vorantrieb. ${ }^{89}$ Er plädiert dabei für „,mediengerechte digitale Editionen“, die „den einzelnen Gegenstand nicht für sich stehen lassen, sondern ihn einbinden in ein Netzwerk von Bezügen“.${ }^{90}$ Hier denkt er etwa an die Verknüpfung des Notentextes mit Tagebüchern, Briefen oder Theaterzetteln. ${ }^{91}$

\subsubsection{Das Edirom-Projekt}

Edirom entstand 2003 am Musikwissenschaftlichen Seminar Detmold/Paderborn. Aus einem Editionsseminar von Gerhard Allroggen und Joachim Veit entstand ein gut einjähriges Projekt, in dem ein „Anzeigeprogramm für die im Rahmen des Seminars bearbeitete Edition von Carl Maria von Webers Klarinettenquintett op. 34“" entwickelt wurde. Diese Edition wurde „2005 als kostenlose Beilage zu Band VI/3 der Carl-Maria-von-Weber-Gesamtausgabe veröffentlicht““.92 Laut Aussage von Johannes Kepper handelt es sich hierbei um die „erste vollständige computerbasierte historisch-kritische Edition eines Werkes der klassischromantischen Periode“. Die Veröffentlichung erfolgte in Form einer CD, die nicht nur den teilweise sogar erweiterten Inhalt des gedruckten Gesamtausgabenbandes enthält, sondern ein Lesartenverzeichnis, das „,zu jeder Einzelanmerkung die zugehörigen Faksimile-Ausschnitte in den editionsrelevanten Quellen“ und den Gesamtausgabentext anzeigt.

Im Umgang mit diesem Meilenstein der Musikedition zeigten sich aber bald zum Teil erhebliche Schwächen hinsichtlich des Datenmanagements und der Nachnutzbarkeit der Daten. Kepper nennt insbesondere folgende Probleme: ${ }^{93}$

- Entwicklung des Anzeigeprogramms auf Basis einer proprietären Software, in diesem Fall Macromedia Director (heute Adobe Director)

- Verwendung von PDF-Plugins, wodurch die Anwendung von der Installation des Adobe Readers abhängig ist

- Software lediglich als Windows-Version (für Windows Vista) verfügbar

\footnotetext{
${ }^{89}$ Kepper 2011, S. 132

${ }^{90}$ Ebenda, S. 134.

${ }^{91}$ Ebenda, S. 133.

${ }^{92}$ Dieses und die folgenden Zitate nach ebenda, S. 162.

${ }^{93}$ Ebenda, S. 163.
} 
So zeigt sich, dass bei dieser ersten Edirom-Version zugunsten arbeitsökonomischer Erwägungen in der Softwareentwicklung und des Versuchs, erst einmal überhaupt auszuloten, was technisch in einer digitalen Edition möglich und sinnvoll ist, Fragen der Nachhaltigkeit außen vor geblieben sind, so dass eine Nutzung dieser Ausgabe auf vielen Rechnern schon heute nicht mehr möglich ist.

In einem von der Deutschen Forschungsgemeinschaft geförderten Projekt zur Entwicklung von Werkzeugen für digitale Formen wissenschaftlich-kritischer Musikeditionen wurde von 2006 bis 2012 an einer neuen quelloffenen Softwarelösung gearbeitet, die nun nicht nur aus einem Anzeigetool, sondern auch aus einem Editor für die Herstellung der digitalen Ausgaben besteht. Die einzelnen technischen Verbesserungen für die Editionsarbeit, die die neue Version gegenüber der ersten Ausgabe besitzt, sind an dieser Stelle nicht weiter relevant. Eine bedeutende Neuerung hinsichtlich der Nachhaltigkeit des Programms besteht nun aber darin, dass hier eine freie Software verwendet wird, bei der eine GNU General Public License zur Anwendung kommt. ${ }^{94}$

In der Folge wurde diskutiert, inwiefern onlinebasierte Publikationsformen für mit Edirom erstellte Editionen einer CD-/DVD-basierten Veröffentlichung vorzuziehen sind. Letztere Lösung wurde dabei vor allem ,,von einigen Bibliotheken und Verlagen favorisiert, da sich die bestehenden Geschäftsmodelle leichter übernehmen lassen und die Sicherung der Inhalte gegen eine unerlaubte Nachnutzung potentiell einfacher ist. Das Edirom-Projekt versucht demgegenüber, durch die Nutzung offener und gut dokumentierter Standards die langfristige Nutzbarkeit der Editionen sicherzustellen. Aus diesem Grund werden die Textbestandteile vollständig in TEI, die Notenteile in MEI codiert. “95

Damit ist das Thema der Verwendung von Datenstandards angesprochen. Hier fordert der Virtuelle Forschungsverband Edirom in einem im April 2016 vorgelegten Positionspapier ${ }^{96}$ „die Verwendung und Einhaltung entsprechender Datenstandards“, ${ }^{97}$ um die Nachnutzbarkeit und den einfachen Austausch von Forschungsdaten und Forschungsergebnissen zu gewährleisten. Die XML-basierten Standards der Text Encoding Initiative (TEI) und der Music Encoding Initiative (MEI) haben sich in den vergangenen Jahren für Text- und Musikeditionen ,,als Basisformate wissenschaftlicher Publikationen“ etabliert. ${ }^{98}$ Für die

\footnotetext{
${ }^{94}$ Ebenda, S. 164 sowie https://github.com/Edirom/Edirom-Online\#license.

${ }^{95}$ Kepper 2011, S. 165 f.

${ }^{96}$ Positionspapier Edirom 2016.

${ }^{97}$ Ebenda, S. 1.

${ }^{98}$ Ebenda, S. 2.
} 
Metadatenauszeichnung von Forschungsdaten wird ferner darauf hingewiesen, dass hier Normdaten wie jene der GND oder der TGN verwendet werden sollten, um die „Anschlussfähigkeit“ der produzierten Daten zu gewährleisten. ${ }^{99}$ Um den Austausch und die interdisziplinäre Vernetzung der Daten sicherzustellen, bedarf es einer Dokumentation der Metadaten und Metdatenschemata, die im Projekt verwendet werden. Daneben betonen die Autoren zugleich die Notwendigkeit der „Dokumentation der Forschungsdaten im engeren Sinne“. Damit meinen sie nicht nur, dass Projektergebnisses im Sinne der DFG-Leitlinien langfristig und qualitätvoll gespeichert und bereitgestellt werden, ${ }^{100}$ sondern dass der gesamte Forschungsprozess soweit offen dokumentiert werden sollte, dass „die Entstehung von Forschungsergebnissen transparenter" wird. Hierbei wird allerdings nicht klar, inwiefern die Autoren von einer damit unterstellten gewissen Intransparenz des Systems der Wissensgenerierung in musikwissenschaftlichen Editionsprojekten ausgehen. Diese Intransparenz scheint zum einem Teil darin zu bestehen, dass der langfristige Wert editorischer Arbeit, der von den Autoren ,in den erarbeiteten Daten selbst“ gesehen wird, in der Praxis oftmals von ,aktuellen ästhetischen und technischen Einflüssen“ bedingt oder sogar überlagert wird. ${ }^{101}$ Um dies zu vermeiden und zu der gewünschten Transparenz zu gelangen, fordern die Autoren für musikwissenschaftliche Editionsprojekte, die über das Akademienprogramm der Union der deutschen Akademien der Wissenschaften durch die Akademie der Wissenschaften und der Literatur Mainz gefördert werden, zukünftig folgende Vorgehensweise: ${ }^{102}$

- Frühzeitige Auswahl eines geeigneten Repositoriums, wo die Forschungsdaten frei zugänglich publiziert werden

- Frühzeitige Einspielung der Projektdaten; bei Langzeitprojekten „spätestens nach einem Drittel der Laufzeit"“

- Etablierung eines Managements der publizierten Forschungsdaten, das nicht nur die Archivierung, sondern auch die Migration in neue Datenformate einschließt

- Das Management der publizierten Forschungsdaten darf dabei nicht als Projektaufgabe angesehen werden, sondern fällt in die Zuständigkeit etablierter Gedächtnisinstitutionen

\footnotetext{
${ }^{99}$ Ebenda.

${ }^{100}$ Leitlinien zum Umgang mit Forschungsdaten, verabschiedet durch den Senat der Deutschen Forschungsgemeinschaft am 30. September 2015.

${ }^{101}$ Positionspapier Edirom 2016, S. 2f.

102 Ebenda.
} 
Damit ist ein kollaboratives Forschungsdatenmanagement umrissen, das nicht nur im Blick auf befristete Editionsprojekte sinnvoll zu sein scheint: Wissenschaftler sind für die Erzeugung der Daten zuständig und achten dabei - gegebenenfalls mit Unterstützung von Forschungsdatenkuratoren - auf die Verwendung und Einhaltung von Datenformaten und Metadatenstandards, die für die Überführung und Einbindung in Repositorien notwendig ist. Das heißt die Wissenschaftler bereiten die Forschungsdaten für die Publikation vor, während das Management der publizierten Daten von Experten auf diesem Gebiet übernommen wird, die auf der Basis der verwendeten standardisierten Formate für die Langzeitarchivierung und dauerhafte Bereitstellung sorgen, um die Nachnutzbarkeit der Forschungsergebnisse eines befristeten Projektes unbefristet zu gewährleisten.

Bei der strategischen Umsetzung dieses Konzeptes sehen die Autoren die Akademien in der Pflicht, einerseits klar Stellung zu dieser arbeitsteiligen Anlage des

Forschungsdatenmanagements zu beziehen, ${ }^{103}$ zweitens technische und juristische Beratungsangebote zu schaffen, um Wissenschaftler bei der Etablierung und Umsetzung einer modernen Verwaltung ihrer Forschungsdaten zu unterstützen, ${ }^{104}$ und drittens sich für eine stärkere Vernetzung der von ihnen geförderten Einzelprojekte einzusetzen. ${ }^{105}$

\subsubsection{Freischütz-Digital ${ }^{106}$}

Das vom Bundesministerium für Bildung und Forschung (BMBF) von 2012 bis 2015 geförderte Projekt ist der praktische Nachfolger des Detmolder Edirom-Projektes. Erstmals wurde hier mit Carl Maria von Webers Freischütz ,der Musik-Codierungsstandard MEI für ein umfangreiches Werk der Common Western Notation angewendet und mit einer TEICodierung der Textanteile verbunden“. ${ }^{107}$ Ziel der Arbeit war es, ,eine wissenschaftlichkritische Edition eines zentralen Werkes der europäischen Musikgeschichte - unter Berücksichtigung unterschiedlicher medialer Ausprägungen im digitalen Medium umzusetzen und mit Aspekten der Genese und Rezeption des Werkes zu verknüpfen. Neben

\footnotetext{
103 Ebenda, S. 3.

${ }^{104}$ Ebenda.

105 Ebenda, S. 4.

${ }^{106}$ Freischütz Digital - Paradigmatische Umsetzung eines genuin digitalen Editionskonzepts,

Gemeinschaftsprojekt der Goethe-Universität Frankfurt, der International Audio Laboratories Erlangen und der Universität Paderborn.

${ }^{107}$ Freischütz Digital. Ziele des Projekts (www.freischuetz-digital.de/index.html).
} 
Notenhandschriften und -drucken werden auch Librettotexte sowie Audioaufnahmen in die Ausgabe, der die Codierungsstandards TEI und MEI zugrunde liegen, integriert.“108 So war es nicht die Absicht des Projektes ,eine Edition im herkömmlichen Sinn, also einen eindeutigen Edierten Text" vorzulegen, sondern einerseits durch eine Codierung der Quellen eine rein deskriptive Erfassung bestehender Sachverhalte und Zusammenhänge zu leisten ${ }^{109}$ und andererseits das Potential neuartiger Editionsmodelle aufzuzeigen, um „künftige Editionsformen zu befördern und mit exemplarischen Untersuchungen zum Aspekt der Varianz weitere Grundlagenforschungen anzuregen“. 110

Mit Blick auf die Verwaltung und Bereitstellung der im Projekt generierten Daten stellt sich die Frage der Nachhaltigkeit damit in zweifacher Hinsicht. Einerseits galt es, die mit den TEIund MEI-Standards codierten Editionstexte selbst anderen Wissenschaftlern für die Nachnutzung zur Verfügung zu stellen, andererseits war es genauso wichtig, die im Projekt entwickelten Workflows für die Codierung nachnutzbar zu machen. ${ }^{111}$ Für die Editionsdaten wird dies über Publikation an mehreren Stellen erreicht. Text-, Musik- und akustische Daten sind in Form von XML-Daten beziehungsweise Audio-Files „einerseits auf den GitHubSeiten zu Freischuetz-Digital, andererseits innerhalb des TextGrid-Repository“ abgelegt. ${ }^{112}$ Die im Projekt entstandenen Werkzeuge, insbesondere die Edirom Online werden entsprechend der oben (Kapitel 3.2.1.) beschriebenen Lizenzbedingungen im Github zur Verfügung gestellt. ${ }^{113}$ Die Beschreibung der Workflows und der entwickelten Demonstratoren wiederum erfolgt über die Projekt-Homepage, die eine umfassende Projektdokumentation bietet. $^{114}$

Insofern versteht das Projekt seine Erfolge auch und vor allem im Blick auf die „in unterschiedlichen Bereichen für weitere Forschungsarbeiten zur Verfügung gestellten Daten und der dokumentierten Workflows“. Denn das ist in der Musikedition paradigmatisch, „dass in allen Teilen des Projekts etablierte Standards benutzt und die Ergebnisse der Community ebenso frei zur Verfügung gestellt werden, um darauf aufbauende oder daran anknüpfende

\footnotetext{
108 Veit 2013.

${ }^{109}$ Kepper 2014, S. 138.

${ }^{110}$ Freischütz Digital. Ziele des Projekts (wie Fußnote 107).

${ }^{111}$ Dokumentation zum Notentext (www.freischuetz-digital.de/documentation-music.html), dort Absatz 2. Zum Editionskonzept.

${ }^{112}$ Dokumentation. Überblick (www.freischuetz-digital.de/documentation.html), dort Absatz 5.

Präsentationskonzept.

${ }^{113}$ https://github.com/Edirom/Edirom-Online.

${ }_{114}$ Dokumentation. Überblick (wie Fußnote 112).
} 
neue Forschungen zu ermöglichen. [...] Insofern ist das Projekt nur ein kleiner Beitrag auf dem Weg zu neuen kooperativen Forschungsformen. “115

\subsubsection{Sarti-Edition ${ }^{116}$}

Mit dem Editionsprojekt A Cosmopolitan Composer in Pre-Revolutionary Europe - Giuseppe Sarti, das von 2013 bis 2016 an der Universität der Künste Berlin unter der Leitung von Christine Siegert und Dörte Schmidt durchgeführt wurde, liegt ein abgeschlossenes Beispiel einer digitalen wissenschaftlichen Edition vor, die an die Entwicklungen der Projekte Edirom und Freischütz-Digital anknüpft. Die Betrachtung ist an dieser Stelle besonders interessant, da diese Edition in Form eines befristeten Drittelmittelprojektes erarbeitet wurde und sich der langfristige Umgang mit den entstandenen Editions- und Forschungsdaten hier schwierig gestaltet. Ziel des Projektes war es, digitale Editionen zweier Opern von Giuseppe Sarti vorzulegen, die Ende des 18. Jahrhunderts in verschiedenen Fassungen an verschiedenen Theatern auf- und wiederaufgeführt worden und deren Fassungsgeschichte sich entsprechend komplex darstellt.

In der Erarbeitung einer digitalen Edition sahen die Projektverantwortlichen die „Möglichkeit, eine große Anzahl verschiedener Fassungen adäquat zu präsentieren“. Das Projekte nutzte dafür die Edirom-Software und den Codierungsstandard der Music Encoding Initiative (MEI). Die besondere Herausforderung der editorischen Codierungsarbeit bestand in der Unterscheidung zwischen ,einer Mikro- und einer Makroebene“, die einerseits auf Ebene der Nummern (Arien und Rezitative) und andererseits auf einer innerhalb dieser Nummern angesiedelten Ebene stattfindet. Der oben beschriebenen Transparenz des wissenschaftlichen Entscheidungsprozesses wurde durch die Einbindung von „Abbildungen der zugrunde liegenden Quellen“ Rechnung getragen werden. Da für die Edition nur eine Auswahl der verfügbaren Quellen herangezogen wurde, sehen die Projektverantwortlichen eine Stärke der digitalen Edition außerdem in der „Möglichkeit, diese jederzeit um weitere Quellen zu ergänzen“. 117

Mit dem letzten Punkt ist bereits die Möglichkeit der Nachnutzung der im Projekt entstandenen Forschungsdaten angesprochen, zu der bisher allerdings keine weiteren Aussagen verfügbar sind. Weder wurden die Daten in einem Repositorium abgelegt, noch

\footnotetext{
115 Ebenda, dort Absatz 2. Arbeitspaket-übergreifende Aspekte.

116 Zum Projekt siehe http://sarti-edition.de/index.html.

117 Zur Projektbeschreibung siehe http://sarti-edition.de/beschreibung.html.
} 
werden sie über die Projekthomepage frei zur Verfügung gestellt. Nicht einmal die Edition selbst ist hier frei einsehbar. Der Versuch sie über die Projekthomepage unter dem Link „Edition“118 aufzurufen, führt derzeit zu einer Authentifizierungsmaske, die die Eingabe eines Benutzernamens und eines Passwortes verlangt. Ein freier Zugriff auf die Edition und deren Forschungsdaten ist damit nicht gewährleistet.

Das Problem, vor dem das Projekt mit seinen Daten hier steht, ist die im Positionspapier des Virtuellen Forschungsverbandes Edirom angesprochene Herausforderung der arbeitsteiligen Verwaltung von Forschungsdaten in Kooperation mit den etablierten Gedächtnisorganisationen. ${ }^{119}$ In dieselbe Richtung weist ein Kommentar von Projektleiterin Dörte Schmidt. Im Tagesspiegel vom 4. November 2015 findet sich ein Text von ihr, in dem sie im Zusammenhang mit einer Aufführung von einer der im Projekt edierten Opern über die Projektarbeit berichtet. Hier schreibt sie zur Nachhaltigkeit der Projektdaten:

„Damit solche Editionen Wirkung zeigen braucht man [...] nicht nur die editorischen Möglichkeiten der digitalen Welt - sondern auch eine Chance auf Beständigkeit des Zugangs zu den daraus resultierenden digitalen Texten, die eben nicht einfach nur digitale Forschungsdaten sind, sondern Kulturgüter überliefern. “120

Der Verweis darauf, dass es sich auch bei digitalen Editionen freilich um Kulturgüter handelt, scheint in dieselbe Richtung zu zielen wie die Überlegungen des Virtuellen Forschungsverbandes Edirom: Gedächtnisinstitutionen, sprich Bibliotheken, Museen und Archive werden hier in der Pflicht gesehen, Angebote zum nachhaltigen Forschungsdatenmanagement vor allem für befristete Forschungs- und Editionsprojekte bereitzustellen.

Dass Gespräche in diese Richtung mit dem an der Bayerischen Staatsbibliothek (seit Januar 2017 in Kooperation mit SLUB Dresden) angesiedelten Fachinformationsdienst Musikwissenschaft (FID Musik) geführt und Überlegungen dahingehend angestellt wurden, die Daten aus dem Sarti-Projekt in der Virtuellen Fachbibliothek Musikwissenschaft ${ }^{121}$ (ViFa Musik) zu archivieren, hat Jürgen Diet im Rahmen des Roundtables Digital Musicology - Wo findet in Zukunft musikwissenschaftliches Wissen statt? bereits am 30. September 2015 in

\footnotetext{
${ }^{118} \mathrm{https}: / /$ sabino.sarti-edition.de sowie https://litiganti.sarti-edition.de.

119 Positionspapier Edirom 2016, S. 3

${ }^{120}$ Schmidt 2015.

${ }^{121}$ www.vifamusik.de
} 
Halle/Saale formuliert. ${ }^{122}$ Die Langzeitarchivierung musikwissenschaftlicher Forschungsdaten solle dabei als Arbeitspaket in den Fortsetzungsantrag für den FID Musik integriert werden. Dieser Antrag wurde im Januar 2017 von der Deutschen Forschungsgemeinschaft bewilligt. Von einem Arbeitspaket zur Verwaltung von Forschungsdaten ist in dem Bericht über den Beginn der Fortsetzungsphase des FID Musik ${ }^{123}$ allerdings keine Rede mehr. Stattdessen finden sich allgemeine Verweise auf ein „musikwissenschaftliches Fach-Repositorium“, das aber eher auf Open-Access-Literatur abzuzielen scheint, und eine Sicherung musikwissenschaftlich relevanter Internetressourcen „über das am Bibliotheksverbund Bayern betriebene Langzeitarchivierungssystem Rosetta“. Ein entsprechendes Angebot zum Abruf der Daten des Sarti-Projekts existiert bis heute nicht.

\subsubsection{Beethovens Werkstatt}

Das Projekt, das seit 2014 in Zusammenarbeit vom Beethoven-Haus Bonn und dem Musikwissenschaftlichen Seminar Detmold/Paderborn durchgeführt und auf 16 Jahre angelegt ist, widmet sich der textgenetischen Erforschung der Werke Ludwig van Beethovens und kombiniert diese mit einer digitalen editorischen Aufbereitung der Forschungsergebnisse in Form der Darstellung von Varianten- und Fassungsbeziehungen von Werkskizzen,

Druckvarianten und Werkbearbeitungen sowie Modelleditionen, die verschiedene im Projekt erarbeitete Editionskonzepte kombinieren. ${ }^{124}$

Methodisch baut das Projekt einerseits auf den Entwicklungen der Projekte Edirom und Freischütz-Digital auf, indem die editorische Arbeit auf der Basis von Codierungen der Werktexte mit dem MEI-Standard geschieht und die Darstellung der Varianten- und Fassungsbeziehungen in der Edirom Online passiert. Zum anderen wird ,in Anlehnung an ein Modell zur Codierung von textgenetischen Prozessen der Text Encoding Initiative (TEI)“ ein spezielles Codierungsmodell für die textgenetische Edition in MEI innerhalb des Projektes entwickelt. Damit leistet das Projekt - ganz im Sinne des von Freischütz-Digital formulierten

\footnotetext{
${ }^{122}$ Sebastian Bolz, Digital Musicology. Wo findet in Zukunft musikwissenschaftliches Wissen statt? Impulsreferate und Podiumsdiskussion, Roundtable-Diskussion im Rahmen der Jahrestagung der Gesellschaft für Musikforschung am 30. September 2015 an der Martin-Luther-Universität Halle-Wittenberg in Halle/Saale (https://lisa.gerda-henkelstiftung.de/digital_musicology_wo_findet_in_zukunft_musikwissenschaftliches_wissen_statt?nav_id=5878).

${ }_{123}$ Jürgen Diet und Barbara Wiermann, Start der Fortsetzungsphase beim Fachinformationsdienst Musikwissenschaft, Pressemeldung der Virtuellen Fachbibliothek Musik vom 16. Februar 2017 (https://kontrovers.musiconn.de/2017/02/16/start-der-fortsetzungsphase-beim-fachinformationsdienstmusikwissenschaft-bsb-muenchen-und-slub-dresden-erstellen-dienstleistungsangebote-fuer-diemusikwissenschaftliche-forschung/).

${ }^{124}$ Zur Projektbeschreibung siehe Cox 2015.
} 
Ziels zur Nachnutzung seiner Daten ${ }^{125}$ - weitere Grundlagenforschung zum Aspekt der Varianz. ${ }^{126}$ Diese Arbeit, so ist die Hoffnung der Projektleiter, wird wiederum neue technische und methodische Fortschritte befördern und insbesondere „der Entwicklung des Codierungsformats der Music Encoding Initiative wertvolle Impulse geben“. ${ }^{27}$

Das Management der in einem solchen Langzeitprojekt anfallenden Daten ist freilich wesentlich komplexer als in zeitlich viel kürzer befristeten Projekten, da hier nicht nur die Aufbereitung der Daten für die externe Nachnutzbarkeit bedacht werden muss, sondern die interne Projektverwaltung dahingehend eine besondere Herausforderung darstellt, als dass hier Daten im Projektzeitraum perspektivisch von verschiedenen Mitarbeitergenerationen erarbeitet werden, die Datenerzeugung über den gesamten Projektzeitraum aber konsistent funktionieren muss. Besonders günstig dürfte sich für das Projekt Beethovens Werkstatt hinsichtlich der Langfristverfügbarkeit seiner Daten auswirken, dass hier mit dem BeethovenHaus Bonn eine Gedächtnisinstitution als Projektpartner beteiligt ist, die sich dem Thema Forschungsdatenarchivierung zumindest organisatorisch - und möglicherweise auch technisch - annehmen kann.

Mit Maja Hartwig und Johannes Kepper haben kürzlich zwei Mitarbeiter von Beethovens Werkstatt einen detaillierten Bericht über das Datenmanagement in digitalen Editionsprojekten vorgelegt, in dem sie vor allem auch mit Blick auf ihr eigenes Projekt über „Die Spuren des Digitalen“ und „die Nachnutzbarkeit digitaler Inhalte“ nachdenken, Probleme benennen und strukturelle und technische Möglichkeiten für deren Lösung vorschlagen. ${ }^{128}$ Dabei ist zunächst interessant, dass sich aus der langjährigen Erfahrung der Autoren in der Arbeit an digitalen Editionsprojekten ${ }^{129}$ eine prinzipiell kritische Sicht auf deren Möglichkeiten und deren Nachhaltigkeit entwickelt hat. Insbesondere merken sie an, dass sich ein „Idealbild einer Digitalen Edition“ etabliert hat, wonach die inhaltliche Codierung der Werkdaten und deren „Verknüpfung mit zugehörigen Faksimiles die [...] Ausgaben [...] so transparent werden lässt, dass alle Entscheidungen des Editors sichtbar und

\footnotetext{
125 Siehe Kapitel 3.2.2. dieser Arbeit.

${ }^{126}$ Cox 2015, S. 14.

${ }^{127}$ Appel 2015, S. 130.

${ }^{128}$ Hartwig 2016.

${ }^{129}$ Maja Hartwig: 2010 bis 2013 wissenschaftliche Hilfskraft im Projekt Digital Music Notation Data Model and Prototype Delivery System, 2013 bis 2014 wissenschaftliche Mitarbeiterin Projekt Digital Research Infrastructures for the Arts and Humanities (DARIAH-DE), seit 2014 wissenschaftliche Mitarbeiterin im Projekt Beethovens Werkstatt; Johannes Kepper: 2003 bis 2012 Mitarbeiter im Projekt Digitale Musikedition (Edirom), seit 2006 aktive Mitarbeit an der Formatentwicklung der Music Encoding Initiative (MEI), 2010 bis 2013 Leitung des Projekts Digital Music Notation Data Model and Prototype Delivery System, 2012 bis 2014 Mitarbeiter im Projekt Freischütz Digital, seit 2014 wissenschaftlicher Mitarbeiter im Projekt Beethovens Werkstatt.
} 
nachvollziehbar gemacht werden könnten“. ${ }^{130}$ Tatsächlich würden aber auch in einer digitalen Edition vielmehr „lediglich die Ergebnisse des editorischen Erkenntnisprozesses angeboten, während die auf dem Weg dorthin stattgefundenen Diskussionen, erwogenen Alternativen und vorgebrachten Argumente weiterhin nicht zwingend Bestandteil der publizierten Edition sind“. Die editorische Arbeit bleibe damit in der Publikation auch weiterhin unsichtbar, so dass eine Nachnutzung nur mit der Einschränkung einer Rekonstruktion der Arbeiten „vom Ergebnis her" möglich sei. ${ }^{131}$

Deshalb beschreiben Hartwig und Kepper im Folgenden eine digitale Projektverwaltung, wie sie etwa im Projekt Beethovens Werkstatt Anwendung findet, und die nicht nur auf die publizierten Editionsdaten - also MEI- und TEI-Codierungen und Bilddaten - fokussiert ist, sondern auch die inhaltliche Konzeption des Projektes, die Verwaltung der verwendeten Terminologien, die Codierungsrichtlinien, die Software und das Projektmanagement mit einschließt.

Als einen wichtigen Bestandteil dieses Dokumentationsprozesses beschreiben Hartwig und Kepper die Versionsverwaltung der Codierungsdaten derart, dass Zwischenstände rekonstruierbar und durch Commit Messages in Form interner Arbeitsnotizen dokumentiert werden. Um den Editionsprozess dabei so transparent wie möglich zu gestalten und die Editionsentscheidungen nachvollziehbar zu machen, müsse der Qualität der Commit Messages eine besondere Beachtung geschenkt werden, da sie wie ein „technisches Protokoll einzelner Arbeitsschritte“ funktionieren. Damit böten sie einen „bislang nicht möglichen Einblick in die tatsächliche Arbeit eines Projekts“. ${ }^{132}$

Der inhaltlich-technische Umgang mit diesen Commit Messages sei verwandt mit der inhaltlich-administrativen Verwaltung der anfallenden Projektarbeiten wie sie im Projektmanagement durch ein Ticketsystem verwaltet werden können. Es regele die Aufgabenverteilung, das heißt die Mitteilung was zu tun sei und wer die Aufgabe zu erledigen habe, es erfasse Arbeits- und Bearbeitungszeiten und erlaube es dem Bearbeiter, Rückfragen beim Auftraggebenden zu stellen. ${ }^{133}$ Der Vorteil eines solchen über ein Ticketsystem organisierten Projektmanagements für das Datenmanagement liegt auf der Hand: Aufgaben,

\footnotetext{
${ }^{130}$ Hartwig 2016, S. 320.

${ }^{131}$ Ebenda, S. $321 \mathrm{f}$.

${ }^{132}$ Ebenda, S. 322f. Es sei dabei nicht nur darauf zu achten, dass die Commit Messages, die die vorgenommenen Änderungen einer Version beschreiben, überhaupt Text enthalten, sondern dass hier auch qualitätvolle Aussagen geschehen, die über den bloßen Hinweis hinausgehen, dass eine Änderung vorgenommen wurde. Eine weitere Herausforderung bestehe darin zu verhindern, dass „Commit Messages von Personen mit größerem technischen Hintergrundwissen einen anderen Inhalt haben als solche von Mitarbeitern ohne dieses Hintergrundwissen“" (S. 323).

${ }^{133}$ Ebenda, S. 324.
} 
Absprachen und bis hin zu Diskussion werden auf diese Weise im digitalen Raum abgebildet und können im Sinne einer transparenten Gestaltung des Forschungsprozesses mit den reinen Forschungsdaten archiviert werden. Dabei sollte freilich dennoch immer das Bewusstsein dafür da sein, das hierbei von einer „,gewissen Lückenhaftigkeit der Daten eines Projektmanagement-Systems“" ausgegangen werden muss, da es in kollaborativen Arbeitsprozessen nie möglich ist, sämtliche Anweisungen und Absprachen rein elektronisch vorzunehmen. ${ }^{134}$ Selbst wenn Besprechungsprotokolle angefertigt und archiviert werden, wird es immer eine gewissen Dimension der persönlichen Kommunikation von Mensch zu Mensch geben, die sich nicht digital abbilden lässt oder aus arbeitsökonomischen Erwägungen nicht abgebildet wird.

In einem Projekt wie Beethovens Werkstatt, das sich neben digitaler Editionsarbeit auch mit Fragen der Grundlagenforschung beschäftigt, gehört zur Projektdokumentation auch die Fixierung der methodischen Arbeit und die Verwaltung des terminologischen Apparates und der Abbildung von dessen Entwicklung. In dem Projekt hat sich dafür die Verwendung eines Wiki-Systems etabliert, in dem die terminologischen Begrifflichkeiten gemeinschaftlich entwickelt, beschrieben und „kontinuierlich revidiert“ werden, bis schließlich „eine hinreichend stabile Fassung gefunden ist“", die dann auf der Projekt-Homepage ${ }^{135}$ mit einer entsprechenden Versionsnummer publiziert und damit für den öffentlichen Diskurs bereitgestellt wird. ${ }^{136}$ Damit folgt das Management der Projektterminologie prinzipiell dem auch für die Forschungsdaten geltenden Data Curation Continuum wie es Andrew Treloar und Cathrine Harboe-Ree 2008 als Modell entworfen haben: In einer sogenannten „Private Research Domain“werden die Daten - beziehungsweise in diesem Fall die terminologischen Begriffe - erzeugt, die dann in dem beschriebenen Wiki als einer „Shared Research Domain“ den Kollegen zur weiteren Bearbeitung bereitgestellt werden. Nach dem Abschluss dieses Bearbeitungsprozesses gelangen die Daten in dem Glossar auf der Projekt-Homepage in die sogenannte „Public Domain“, die einen (vorläufigen) Abschluss der Forschungstätigkeit darstellt. $^{137}$

Mit Blick auf die Fixierung der Codierungsrichtlinien weisen Hartwig und Kepper darauf hin, dass es hier nicht allein um die Darlegung des technischen Rahmens der Edition geht, sondern dass diese ,in ihrer Funktion weitgehend den traditionellen Editionsrichtlinien

\footnotetext{
${ }^{134}$ Ebenda S. 325.

135 Beethovens Werkstatt. Glossar (http://beethovens-werkstatt.de/glossary).

${ }^{136}$ Hartwig 2016, S. 326.

${ }^{137}$ Zum Data Curation Continuum von Andrew Treloar und Cathrine Harboe-Ree siehe Rümpel 2011, S. 29f.
} 
entsprechen“. ${ }^{138}$ Da diese im Verlauf - gerade bei einem Langzeitprojekt - einem kontinuierlichen Wandel unterliegen, gilt es nach technischen Möglichkeiten zu suchen, die es erlauben, Inkonsistenzen zu vermeiden. Der Einsatz der Schemasprache ODD ${ }^{139}$ wird hierfür als „die beste Möglichkeit beschrieben“, da sich die Datenformate TEI und MEI hier den projektspezifischen Erfordernissen entsprechend anpassen lassen, ,so dass nur die in der Edition benötigten und zu nutzenden Funktionen zur Verfügung stehen“. Die saubere Abbildung der Codierungsrichtlinien in ODD ist dabei eine wichtige Voraussetzung für die Nachnutzbarkeit der Forschungsdaten. ${ }^{140}$

Unabhängig von den reinen Daten bedarf es für die vollständige Rekonstruktion und Nachnutzung eines Projektes auch der hierbei verwendeten Softwareumgebung. Zwar werden die Forschungsdaten im Projekt Beethovens Werkstatt und in allen weiteren MEI- bzw. Edirom-basierten digitalen Editionsprojekten auf XML-basierenden Standards dokumentiert, dennoch ist allein mit diesen Daten, das erarbeitete Editionsvorhaben nicht vollständig wiederherstellbar. Ob es aber möglich ist, in gleicher Weise wie für die Daten ein nachhaltiges und archivierbares Konzept auch für die Software eines Projektes zu ermöglichen, ,ist aus heutiger Perspektive schwer zu beurteilen“. ${ }^{141}$

„Die beste Strategie eines Editionsprojekts ist in dieser Hinsicht sicherlich der Einsatz von Open-Source-Software, die - ebenso wie die Daten - möglichst ausführlich zu dokumentieren ist. Damit kann ggf. auch die Benutzeroberfläche der Edition rekonstruiert werden.“

Damit stellt sich für musikwissenschaftliche Editionsprojekte ein ähnliches Problem wie es aus der Archivierung von Computerspielen bekannt ist, auch wenn sich die Sachverhalte dort freilich viel komplexer darstellen. ${ }^{142}$

Bei Beachtung dieser Säulen des Projekt- und Datenmanagements ergibt sich für ein digitales Projekt ein durchaus hoher Grad an Nachnutzungspotential. Nachvollziehbar erscheint aber auch der Hinweis der Autoren, dass ,,in vielen bisherigen Editionsprojekten die Fülle an Informationen mitunter als problematisch erfahren wurde“, insbesondere ,wenn bei der

\footnotetext{
${ }^{138}$ Hartwig 2016, S. 327

139 „One Document Does it all“ (http://wiki.tei-c.org/index.php/ODD).

${ }^{140}$ Hartwig 2016, S. 328.

${ }^{141}$ Dieses und das folgende Zitat ebenda, S. 329.

142 Siehe Huth 2010.
} 
Auswertung dieser Informationen der mögliche Erkenntnisgewinn den dafür nötigen Zeitaufwand nicht mehr rechtfertigt"،. ${ }^{143}$

\subsection{Digitale Erschließung musikalischer und musikgeschichtlicher Quellen}

Haben Bibliotheken relativ frühzeitig damit begonnen ihre Buchbestände in digitalen Onlinekatalogen zu erfassen, so ist die Erfassung von Handschriftenbeständen erst relativ spät gefolgt. Dieser Umstand hängt sicher ganz wesentlich mit dem Missverhältnis in der Nutzung der beiden recht unterschiedlichen Bestandsgruppen zusammen. Während die Buchbestände von einer breiten Leserschaft genutzt und recherchiert werden wollen, sind Handschriftenbestände für einen viel kleineren, oft gut informierten Nutzerkreis interessant. Für diese Nutzer ist die Erschließung von zentralen Bestandsgruppen in gedruckten Katalogen erfolgt, die Bestands- und auch disziplinspezifisch recht unterschiedliche Erschließungstiefen aufweisen.

Das zentrale Nachweisinstrument für Musikhandschriften und alte Musikdrucke ist das 1952 in Paris gegründete Répertoire International des Sources Musicales (RISM). Das kollaborative Netzwerk aus 36 nationalen Arbeitsgruppen arbeitet „,mit dem Ziel, die weltweit überlieferten Quellen zur Musik umfassend zu dokumentieren“. ${ }^{144}$ Seit 2010 ist das Verzeichnis als kostenloser Onlinekatalog (https://opac.rism.info $)$ - in Kooperation von RISM, der Bayerischen Staatsbibliothek und der Staatsbibliothek zu Berlin - frei zugängig und verzeichnet inzwischen über eine Million Datensätze zu historischen Notenbeständen aus aller Welt. ${ }^{145}$ Die Staatsbibliothek zu Berlin übernimmt in dem Kooperationsverbund das Hosting des Erschließungssystems, das Ende 2016 von Kallisto auf Muscat umgestellt wurde. Die Bayerische Staatsbibliothek hostet das Präsentationssystem, also den RISM-OPAC, und ist damit für die langfristige Sicherung der hier gespeicherten musikwissenschaftlichen Forschungsdaten zuständig. Diese Daten „,werden als Linked Open Data im RDF-Format und als Open Data im MARC-XML-Format bereitgestellt ${ }^{\star 146}$ und bieten damit gute

\footnotetext{
${ }^{143}$ Hartwig 2016, S. 328.

${ }^{144}$ Siehe Kapitel 1.1. dieser Arbeit sowie https://rism.info/de/organization.html und https://rism.info/de/workinggroups.html.

${ }^{145}$ Rita Benton und Jennifer A. Ward, Répertoire International des Sources Musicales, in: GMO (https://doi.org/10.1093/gmo/9781561592630.article.23216).

${ }^{146}$ Bayerische Staatsbibliothek engagiert sich bei internationalen Projekten zum Nachweis von musikalischen und musikikonographischen Quellen, Pressemitteilung der Bayerischen Staatsbibliothek vom 17. August 2016 (https://www.bsb-muenchen.de/fileadmin/pdf/presse/2016/pm2016_koop_rism_ridim.pdf).
} 
Voraussetzungen für die wissenschaftliche und insbesondere interdisziplinäre Nachnutzung im Semantic Web.

Da die Erschließung historischer musikalischer Quellenbestände - die heute projektübergreifend fast überall innerhalb des RISM erfolgt - zu den Hauptaufgaben musikwissenschaftlicher Forschungsarbeit zählt, sollen im Folgenden mit dem KoFIMProjekt der Staatsbibliothek zu Berlin und dem Digitalisierungs- und Erschließungsprojekt „Sammlung Manfred Gorke“ des Bach-Archivs Leipzig wenigstens zwei aktuelle Quellenerschließungsprojekte vorgestellt werden. Mit dem Online-Portal zur RepertoireErforschung des Thomanerchores soll sich abschließend noch einem Erschließungsprojekt gewidmet werden, das sich mit der Auswertung von Repertoireverzeichnissen einer weiteren Gattung musikhistorischer Quellenbestände annimmt.

\subsubsection{KoFIM Berlin - Kompetenzzentrum Forschung und Information Musik}

Mit der Etablierung eines Kompetenzzentrums Forschung und Information Musik hat die Staatsbibliothek zu Berlin seit 2012 ausgehend von der Förderinitiative „Wissenschaftliche Literaturversorgungs- und Informationssysteme“ der Deutschen Forschungsgemeinschaft den Ausbau ihrer Musikabteilung von einer ,ursprünglich reinen Dienstleistungsbibliothek zu einem modernen Forschungszentrum“ vorangetrieben. ${ }^{147}$ Aufgabe des auf sechs Jahre angelegten Projektes ist die Tiefenerschließung von einem Teil des autographen Musikhandschriftenbestandes; und zwar jenes Teils der Sammlung, der noch nicht durch neuere Kataloge in Buchform, sondern lediglich in Zettelform erschlossen vorliegt. ${ }^{148}$ Unter Tiefenerschließung versteht das KoFIM dabei die vernetzte und Datenbank-gestützte Grundlagenforschung an den eigenen Beständen erstens zur Klärung von Provenienzfragen, zweitens zur Schreiberforschung und zur Dokumentation der Schriftformen von Komponisten und Kopisten und drittens zur Dokumentation und Klassifikation von Wasserzeichen, die die Papiere des Musikalienbestandes in sich tragen. In dem als Pilotphase angelegten Projekt sollen neue Methoden der wissenschaftlichen Dokumentation von Musikhandschriftenmerkmalen erarbeitet und dabei „Arbeitsroutinen und Geschäftsgänge für die Etablierung eines sogenannten ,Catalog enrichment‘ auf dem Gebiet der digitalen Dokumentation von Schreiberhänden und von Wasserzeichen neu entwickelt" werden. ${ }^{149}$

\footnotetext{
${ }^{147}$ Eckhardt 2013, S. $12 \mathrm{f}$.

148 Rebmann 2016, S. 14.

149 Ebenda, S. 17.
} 
Die Erschließung der Musikalienbestände - es handelt sich um Quellen des 17. bis frühen 19. Jahrhunderts - erfolgt dabei über das RISM nach den dort definierten einheitlichen und standardisierten Katalogisierungsrichtlinien. Hierbei spielt für KoFIM insbesondere die Erforschung und Dokumentation der Provenienzen eine zentrale Rolle. RISM bietet dafür eine eigene Normdatenbank für Personen und Körperschaften, die für den Endnutzer zwar nicht - wie etwa die GND - separat abrufbar ist, deren Einträge aber auf bibliographischer Ebene der Datensätze eingebunden und so über die Suchmasken auch recherchierbar sind. ${ }^{150}$ Seit der Umstellung von Kallisto auf Muscat bietet die Erfassungssoftware außerdem eine Schnittstelle zum Import von Personen-Normdaten aus dem Virtual International Authority File (VIAF). ${ }^{151}$ Problematischer stellt sich die Dokumentation von Provenienzmerkmalen dar. Die Beschreibung von Einband und Material ist über ein Freitextfeld möglich, dem keinerlei Standardisierungen zugrunde liegen. Durch die prinzipielle Dreisprachigkeit des RISM (deutsch, englisch, französisch) wird die Auffindbarkeit und Nachnutzung hier hinterlegter Daten zusätzlich erschwert. Möglichkeiten eines Catalog enrichments in Form von Bilddateien von Provenienzmerkmalen werden ebenfalls nicht genutzt, normiertes Vokabular findet keine Anwendung. Einen perspektivischen Lösungsansatz sieht Julia Neumann in der Anbindung des an der Herzogin Anna Amalia Bibliothek in Weimar entwickelten Thesaurus für Provenienzforschung T-PRO an das RISM. Dies scheint nicht nur im Blick auf eine interdisziplinäre Nachnutzung der musikwissenschaftlich erschlossenen Provenienzdaten sinnvoll, sondern bietet sich aufgrund der ebenfalls dreisprachigen Anlage des T-PRO in Deutsch, Englisch und Französisch für das RISM geradezu an. ${ }^{152}$

Fortgeschrittener ist in dieser Hinsicht die digitale Dokumentation von Schreiberhänden. Hier ist der Anspruch des KoFIM, unter der Beachtung der Auswahl charakteristischer Schriftmerkmale und unterschiedlicher Schriftstadien einen Referenzbestand an Digitalisaten für die quellenbasierte Schreiberforschung zur Verfügung zu stellen. Hier besteht zwar zunächst dasselbe Problem wie bei der Verankerung der Provenienzbeziehungen, nämlich dass die entsprechenden Informationen nicht separat abrufbar sind, sondern über die Personendatensätze des RISM verlinkt sind, die nur über den Umweg der Quellendatensätze erreichbar sind. Dennoch führen gezielte Katalogrecherchen nach Schreibern hier sehr schnell zu den entsprechenden digitalen Referenzdaten. ${ }^{153}$

\footnotetext{
${ }^{150}$ Neumann 2016, S. 74.

${ }^{151}$ Willkommen Muscat, News-Feed des RISM vom 14. November 2016 (https://rism.info/de/new at_rism/2016/11/14/welcome-muscat.html).

152 Neumann 2016, S. 75-78.

${ }^{153}$ Eckhardt 2013, S. 15.
} 
Eine weitere Herausforderung in der Verwaltung der Erkenntnisse der Schreiberforschung stellt das Zusammenführen der Ergebnisse isoliert durchgeführter Schreiberuntersuchungen dar. Dieselben Musikalienkopisten tauchen in den unterschiedlichsten Forschungskontexten auf und werden in unabhängig voneinander entstehenden Publikationen beschrieben. Ihre Identifizierung und Benennung mit Nomenklaturen folgt dabei keinen standardisierten Regeln, so dass die Ergebnisse nicht vergleichbar und kontextualisierbar sind. Das KoFIM bemüht sich um die digitale Zusammenführung der Teilergebnisse im RISM. Hier werden die unterschiedlichen Nomenklaturen eines anonymen Kopisten durch gegenseitige Verweise in den Namensdatensätzen vereint und schließlich neue normierte Ansetzungsformen eingeführt. ${ }^{154}$

Die sinnvolle Verwaltung und Erschließung des wachsende Datenbestandes an digitalen Schriftproben, der im KoFIM entsteht, ist perspektivisch nur sinnvoll über die Etablierung eines Klassifizierungssystems für Musikerhandschriften, noch besser wohl aber mit den Techniken einer automatisierten Schreibererkennung auf Basis der Bilddaten möglich. Denn ohne eine zuverlässige Bestimmung identischer Schreiberhände ließen sich auch im digitalen Dokumentationsbereich Dubletten und die Vergabe doppelter Nomenklaturen für anonyme Schreiber nicht vermeiden. Sollte dies aber gelingen, und der Pilotcharakter des KoFIM Vorbildwirkung auch auf andere Bibliotheken entfalten, könnte aus diesem Ansatz durch kooperative Erschließung im besten Fall eine „breit angelegte virtuelle Schriftproben-Kartei“ entstehen. ${ }^{155}$ Die Sicherung der hier abgelegten Daten und deren Bereitstellung wären über das RISM jedenfalls sinnvoll und nachhaltig abgedeckt.

Neben der Provenienz- und Schreiberforschung stellt die digitale Dokumentation der Wasserzeichen in den Handschriften des Autographenbestandes die dritte Säule der Grundlagenforschung des KoFIM dar. Auch hier findet die Technologie des RISM Anwendung, indem ein Referenzbestand an Bilddaten ${ }^{156}$ aufgebaut wird, der durch Verlinkungen in den Personendatensätzen recherchierbar ist. Die fehlende Erfahrung des RISM im Umgang und insbesondere der Klassifikation dieser Bilddaten löst das KoFIM durch die Kooperation mit dem Wasserzeicheninformationssystem WZIS am Landesarchiv Baden-Württemberg. Dabei wird die Erfassung der Daten im WZIS durch das KoFIM vorgenommen, indem ,zunächst die verbale Beschreibung sämtlicher ermittelter

\footnotetext{
${ }^{154}$ Ebenda, S. 18f.; sowie Schwinger 2016, S. 126.

155 Schwinger 2016, S. 128.

${ }^{156}$ Die Erzeugung der Bilddaten erfolgt dabei mittels einer Thermographiekamera, die die Manuskripte mit Infrarotstrahlung durchleuchtet. Siehe Eckhardt 2013, S. 16.
} 
Wasserzeichen einer Handschrift im RISM/Kallisto“ erfolgt, dann wird das Digitalisat in WZIS eingepflegt und abschließend erfolgt eine Kreuzverlinkung zwischen dem RISMPersonendatensatz und dem WZIS-Digitalisat. ${ }^{157}$ Die Kooperation mit WZIS ermöglicht dabei die Suchbarkeit und überhaupt erst die Sichtbarkeit der digitalisierten Wasserzeichen, indem dort neben der Präsentation auch die Vorhaltung der Digitalisate und der dazugehörigen Metadaten stattfindet. ${ }^{158}$ Im Gegensatz zu der ,digitalen Schreiberkartei“, die das KoFIM erstellt, sind der interdisziplinäre Ansatz und die Öffnung der Arbeit über die fachspezifische RISM-Datenbank hinaus für die Wasserzeichendokumentation der sinnvollste Ansatz. Die Daten, die im Zuge musikwissenschaftlicher Grundlagenforschung gewonnen werden, fließen so in einen „fächerübergreifenden Datenpool“, wodurch zugleich eine „Fragmentierung von Rechercheangeboten“" vermieden wird. ${ }^{159}$ Die Nachnutzung der vom KoFIM erzeugten Daten im Sinne eines verantwortungsvollen Forschungsdatenmanagements ist damit unter Beachtung disziplinspezifischer Anforderungen und interdisziplinärer Möglichkeiten nicht nur gegeben, sondern durchaus dazu geeignet, auf Basis der drittmittelfinanzierten sechsjährigen Pilotphase Modellcharakter zu entwickeln.

\subsubsection{Sammlung Manfred Gorke - Digitalisierung und Erschließung ${ }^{160}$}

Dass kooperative Lösungsansätze bei der wissenschaftlichen Erschließung von lokalen Handschriftenbeständen ideale Möglichkeiten bieten, zeigt auch das derzeit am Bach-Archiv Leipzig durchgeführte Digitalisierungs- und Erschließungsprojekt „Sammlung Manfred Gorke“. Die Musikaliensammlung aus dem Familienbesitz des Eisenacher Sparkassenangestellten Manfred Gorke umfasst 700 Objekte zur sächsisch-thüringischen Musikgeschichte des 17. bis 19. Jahrhunderts und wird seit 1952 im Bach-Archiv aufbewahrt. ${ }^{161}$ Auf eine erste handschriftliche Erschließung des Bestandes in den 1930er Jahren folgte 1977 ein gedruckter Katalog. ${ }^{162}$ Im Rahmen der Projektarbeiten an Bach digital ${ }^{163}$ hatte sich am Bach-Archiv in den letzten Jahren zunehmend die Frage nach der Digitalisierung der in der Sammlung Gorke enthaltenen Bach-Handschriften gestellt, und

\footnotetext{
${ }^{157}$ Ebenda, S. 17.

${ }^{158}$ Ebenda, S. 17; sowie Eckhardt 2016, S. 180.

${ }^{159}$ Ebenda, S. 193.

${ }^{160}$ Das Folgende, soweit nicht anders angegeben, als Erfahrungsbericht des Autors dieser Arbeit, der als wissenschaftlicher Mitarbeiter im Forschungsinstitut und wissenschaftlicher Referent der Forschungsbibliothek des Bach-Archivs dort für die Koordination des Projektes zuständig ist.

${ }^{161}$ Bärwald/Meyer 2016.

162 Schulze 1977.

${ }^{163}$ Siehe Kapitel 1.1. und 2.1. dieser Arbeit.
} 
damit auch die Frage nach dem Umgang mit dem Rest des Bestandes. Dieser überliefert auch jenseits der Bachiana einzigartige Musikalien, deren Digitalisierung und Erschließung sowohl unter konservatorischen als auch wissenschaftlichen Gesichtspunkten sinnvoll ist, aber durch die Projektrichtlinien von Bach digital nicht abgedeckt wird.

Da das Bach-Archiv nicht in der Lage gewesen wäre, die Digitalisierung und Erschließung der Sammlung sowohl wirtschaftlich als auch personell selbstständig zu leisten und sie technisch umzusetzen, wurde nach einem leistungsfähigen Projektpartner gesucht, der schließlich in der SLUB Dresden und dem von ihr koordinierten

Landesdigitalisierungsprogramm für Wissenschaft und Kultur des Freistaates Sachsen ${ }^{164}$ gefunden wurde.

Ausgangspunkt der Projektarbeiten war zunächst die Erschließung sämtlicher Objekte der Sammlung im Verbundkatalog des Südwestdeutschen Bibliotheksverbundes. Die Digitalisierung erfolgte anschließend in der Digitalisierungssoftware Kitodo. Ein erster Teil der erzeugten Daten wird inzwischen über die Digitalen Sammlungen der SLUB Dresden bereitgestellt ${ }^{165}$ und auf deren Servern gehostet. Die Strukturierung der Digitalisate erfolgte wieder im Bach-Archiv, da die Frage nach der Tiefe der Strukturebene sehr von der wissenschaftlichen Bewertung des jeweiligen Objektes abhängt, wobei der Inhalt der Sammlung extrem inhomogen ist und neben Musikalien auch Briefe, Freundschaftsalben und archivalische Quellen enthält.

Im Anschluss an die Digitalisierung soll schließlich noch eine Tiefenerschließung der Musikalien erfolgen, die dann in der Arbeitsstelle des RISM ebenfalls in Dresden stattfindet, wobei die Digitalisate aus den Digitalen Sammlungen der SLUB Dresden in den RISMKatalogisaten verknüpft werden.

Entscheidend ist bei diesem Projekt neben der finanziellen Unterstützung durch das Landesdigitalisierungsprogramm, dass hier mit der SLUB Dresden eine Bibliothek als zentraler Koordinator für die Akkumulation wissenschaftlicher Forschungsdaten eintritt und schließlich auch die Archivierung und langfristige Bereitstellung über ein eigenes Langzeitarchivierungssystem anbietet. Gerade für kleinere Gedächtnisinstitutionen wie das Bach-Archiv bietet das die Möglichkeit, einerseits seine Bestände digital zu sicher, zweitens das Wissen um diese Bestände digital zu verwalten und schließlich beides gemeinsam Bestand und Wissen - in dauerhaften und nachhaltigen Forschungsumgebungen der

\footnotetext{
${ }^{164} \mathrm{http}: / / \mathrm{www}$. slub-dresden.de/sammlungen/landesdigitalisierungsprogramm.

165 https://digital.slub-dresden.de/kollektionen.
} 
Öffentlichkeit zu präsentieren und für die weitere wissenschaftliche Auseinandersetzung bereitzustellen.

\subsubsection{Repertoire-Erforschung des Thomanerchores}

Am Musikwissenschaftlichen Institut der Universität Leipzig wurde in Kooperation mit der Abteilung Automatische Sprachverarbeitung des Instituts für Informatik der Universität Leipzig ein Projekt betrieben, dass sich der EDV-gestützten Dokumentation des Repertoires des Leipziger Thomanerchors für die Jahre 1808 bis 2008 gewidmet hat. ${ }^{166}$ Das Projekt wurde anlässlich des Jubiläums zum 800-jährigen Bestehen des Thomanerchores (2012) initiiert und von 2009 bis 2015 durchgeführt. Daher sollte sich das entstandene Portal ${ }^{167}$ nicht nur an Musikwissenschaftler, sondern auch an Laien richten, „die durch das Thomaner-Jubiläum 2012 und die damit verbundene Öffentlichkeitsarbeit auf das Portal aufmerksam gemacht werden". 168

Die Projektarbeit versteht sich als Grundlagenforschung an den historischen Quellen, die die Datenbasis der Dokumentation darstellen. Dabei handelt es sich einerseits um das Motettenbuch des Chores, das die Aufführungen in den Samstagsmotetten für die Jahre 1811 bis 1905 verzeichnet, ferner wurden der Sammelbestand an gedruckten Programmzetteln aus dem Archiv des Chores sowie als komplementäre Quelle die Konzertankündigungen des Leipziger Tageblattes ausgewertet. ${ }^{169}$

Die aus diesen Quellen gewonnenen Daten fließen über eine Ruby on Rails Anwendung in eine MySQL-Datenbank, wo sie in einer Topic Map basierten Ontologie angelegt werden. Bei Eingabe werden die einzelnen Topics über in der Datenbank hinterlegte Normdateien - zur Anwendung kommen der Datensatz der Personennormdatei PND, der Gemeinsamen Körperschaftsdatei GKD und der Einheitssachtiteldatei EST - abgeglichen und gegebenenfalls dem Normdatensatz nach den Vorgaben der RAK-Musik und RAK-WB hinzugefügt. Aus den einzelnen Topics wie Personen, Institutionen, Aufführungsorten,

\footnotetext{
${ }^{166}$ Eine ausführliche Beschreibung der musikwissenschaftlich-inhaltlichen Aspekte des Projektes bieten Loos 2012 und Götz 2016, eine nähere Darstellung der technisch-strukturellen Inhalte findet sich bei Stöck 2012. ${ }^{167} \mathrm{http}$ ://thomaner.topicmapslab.de - Die Projektseite ist inzwischen (Stand: 3. März 2021) nicht mehr erreichbar. Die Projektdaten sind seit 2020 über musiconn.performance (https://performance.slub-dresden.de) recherchierbar.

168 Stöck 2012, S. 37.

${ }^{169}$ Loos 2012, S. 220.
} 
Werknamen, Gattungen, Besetzungen und Textquellen werden kombinierte Datensätze zu den Kompositionen und den einzelnen Auftritten akkumuliert. ${ }^{170}$

Mit Blick auf die Nachnutzung der erarbeiteten Datenbank beschreibt Stöck die Verwendung von Topic Maps dahingehend als Vorteil, dass dadurch „die schnelle Adaption des Portals für zukünftige, ähnlich gelagerte Projekte, die eine gemeinsame Datenbasis zur Repertoireforschung haben“", möglich ist. ${ }^{171}$ Insofern könnte das Portal Modellcharakter entwickeln, so dass auch andere Arbeiten zur Repertoireforschung ,weit über den lokalen Leipziger Rahmen“ hinaus die hier entwickelte Infrastruktur nachnutzen könnten. ${ }^{172}$ Die Sicherung der angelegten Forschungsdaten erfolgt über den „Funktionsumfang für Transaktionssicherheit und Backup-Sicherheit“ innerhalb des Relational Database Management Systems. Damit ist aber noch nichts über die langfristige Archivierung der Projektdaten gesagt; und hierüber finden sich auch keine Aussagen der Projektverantwortlichen. Die Homepage des Rechercheportals jedenfalls wird über die Universität Leipzig gehostet. Daher erscheint es sinnvoll, dass die Universität - die über die Abteilung Automatische Sprachverarbeitung beispielsweise eines der deutschen Clarin-D Zentren betreibt ${ }^{173}$ - perspektivisch auch für die Langzeitarchivierung der im Backend von MySQL gespeicherten Forschungsdaten sorgen sollte.

\section{Fazit}

Das Thema Forschungsdatenmanagement spielt im musikwissenschaftlichen Arbeiten der Historischen Musikwissenschaft zunehmend eine wichtige Rolle, da immer mehr Forschungsarbeiten mit digitalen Werkzeugen erfolgen und dabei digitale Daten entstehen, für deren Archivierung es noch keine disziplinspezifisch etablierten Lösungen gibt. Dies ist gerade vor dem Hintergrund dessen kritisch zu betrachten, dass die musikwissenschaftlichen Editions- und Quellenerschließungsprojekte durchaus disziplinspezifische Forschungsdaten produzieren. Diese werden zurzeit in den Langzeitarchivierungsinfrastrukturen von Universitäten wie etwa den Clarin-D Zentren und bei großen überregionalen Bibliotheken wie der Staatsbibliothek zu Berlin, der Bayerischen Staatsbibliothek oder Sächsischen

\footnotetext{
170 Stöck 2012, S. 35 und 37; sowie Götz 2016, S. 6.

171 Stöck 2012, S. 37.

172 Götz 2016, S. 9.

${ }^{173} \mathrm{https}$ ://repo.clarin.informatik.uni-leipzig.de/de.
} 
Landesbibliothek - Staats- und Universitätsbibliothek Dresden gehostet. Dieser projekt- und nicht disziplinspezifische Umgang scheint dabei typisch für die Geisteswissenschaften zu sein und folgt ähnlichen Regeln wie die Datenablage im analogen Raum (derart dass die betreuenden oder übergeordneten Gedächtnisinstitutionen mehr oder weniger automatisch in die Rolle der Archivstelle von Forschungsdaten geraten). Diese Situation hat freilich einerseits damit zu tun, dass sich auf Ebene der Fachgesellschaften - konkret bei der Gesellschaft für Musikforschung - erst nach Aufforderung durch die Deutsche Forschungsgemeinschaft zur Positionierung zu deren Leitlinien zum Umgang mit Forschungsdaten ein akuter Handlungsbedarf zur Auseinandersetzung mit Fragen des Forschungsdatenmanagements ergeben hat. Der damit eingeleitete Prozess ist derzeit noch nicht abgeschlossen. Neben diesem äußeren Grund ist es aber andererseits vor allem die Rolle der Historischen Musikwissenschaft als Small Science ${ }^{174}$ und damit das Fehlen hochgradig datenintensiver Arbeitsfelder, die dazu geführt hat, dass ein breiteres Problembewusstsein zum Thema Forschungsdatenmanagement sich lange Zeit überhaupt nicht entwickelt hat und im Fach auch immer noch nicht flächendeckend angekommen ist.

Weniger die Arbeit mit digitalen Werkzeugen, sondern vielmehr die Notwendigkeit, eigene neuartige Forschungsansätze und damit verbundene Forschungsdaten in den Kontext der Digital Humanities im Sinne einer Big Science einzubringen ${ }^{175}$ - das heißt eigene Forschungen für die interdisziplinäre Nachnutzung anzubieten wie auch selbst von anderen interdisziplinären Forschungsarbeiten zu profitieren -, hat schließlich dazu geführt, dass sich (und das ist dezentral geschehen) Workflows und fachspezifische Datenstandards etabliert haben, die im interdisziplinären Forschungskontext bestehen können.

Mit Blick auf die in Kapitel 3 beschriebenen Projekte, die hinsichtlich der Entwicklung von Referenzmodellen ${ }^{176}$ zum digitalen Arbeiten und damit auch im Blick auf das betriebene Forschungsdatenmanagement weitgehend Erfolgsgeschichten sind, scheint für die infrastrukturelle Gestaltung eines musikwissenschaftlichen Forschungsdatenmanagements eine deskriptive Positionsbestimmung gegenüber einer normativen Modellierung grundlegende Vorteile zu haben. Gerade mit Blick auf die Prinzipien einer Small Science sind es die vielen projektspezifischen Lösungen, die ganz unterschiedlichen wissenschaftlichen Anforderungen geschuldet sind und die gar nicht sinnvoll in einer einzigen technischen

\footnotetext{
174 Siehe dazu näher in Kapitel 2.2. dieser Arbeit.

175 Siehe in Kapitel 3.1. dieser Arbeit.

${ }^{176}$ Siehe die Projekte Edirom (Kapitel 3.2.1.) und KoFIM (Kapitel 3.3.1.) oder die Datenbank zum Repertoire des Thomanerchores (Kapitel 3.3.3.).
} 
Infrastruktur zusammengeführt werden könnten. Vielmehr geht es darum, stets projektspezifisch nach Lösungen zu suchen, die den konkreten Bedürfnissen nach interdisziplinärer Vernetzung gerecht werden - zu nennen wären hier beispielsweise die grundlegenden strukturellen Unterschiede in der Vernetzung des KoFIM mit dem WZIS einerseits und des Edirom-Projektes mit der Informatik andererseits.

Die Erarbeitung eines fachspezifischen Konzepts zum Umgang mit Forschungsdaten wie es die Deutsche Forschungsgemeinschaft fordert, ist damit insbesondere hinsichtlich der Punkte „Datentypen“, „disziplinspezifische Standards“ und „Wahl geeigneter Repositorien“"177 _ selbst für einen so eng abgesteckten Bereich wie die in dieser Arbeit betrachtete musikwissenschaftliche Editionsarbeit und die digitale Erschließung musikalischer und musikgeschichtlicher Quellen - aufgrund der faktischen Heterogenität schwierig. Selbst bei Fragen wie jener nach der zeitnahen Bereitstellung der Forschungsdaten, zeigen sich ganz unterschiedliche Dringlichkeiten: während wissenschaftliche Erschließungsdaten zu Quellenbeständen in der Regel möglichst zeitnah (nach-)genutzt werden wollen, ist eine vergleichbare Dringlichkeit bei Editionsprojekten nicht zu erkennen.

Besser Schematisieren lässt sich dagegen der Aspekt der „Langfristigen Sicherung“. Hier hat sich gezeigt - und auch das ist typisch für eine Small Science -, dass fachspezifische technische Infrastrukturen keine besondere Rolle spielen, sondern musikwissenschaftliche Forschung hinsichtlich ihrer Langzeitarchivierung immer auf Kooperationen angewiesen ist. In der Regel sind es die oben genannten Gedächtnisinstitutionen, die hier eine zentrale Rolle spielen - sie übernehmen diese Rolle einerseits aktiv, ${ }^{178}$ andererseits wird sie von den Forschenden auch ganz konkret eingefordert. ${ }^{179}$

Vor dem Hintergrund dieser guten und sinnvollen Zusammenarbeit zwischen Bibliotheken und Forschenden ist es völlig unverständlich, warum - entgegen anders lautender Bekundungen $^{180}$ - gerade der Fachinformationsdienst Musikwissenschaft (FID Musik) hier bisher keine aktivere Rolle einnimmt und beispielsweise ein zentrales Angebot gerade für die Archivierung von Forschungsdaten aus zeitlich befristeten Drittmittelprojekten aufbaut.

Dieses müsste nicht nur auf die Archivierung von Daten aus dem engeren Themenbereich der Digitalen Musikwissenschaft abzielen, sondern könnte viel mehr das Problembewusstsein für

\footnotetext{
${ }^{177}$ Leitlinien zum Umgang mit Forschungsdaten, verabschiedet durch den Senat der Deutschen Forschungsgemeinschaft am 30. September 2015 (http://www.dfg.de/download/pdf/foerderung/antragstellung/forschungsdaten/richtlinien_forschungsdaten.pdf).

178 Wie beispielsweise die SLUB Dresden mit dem Landesdigitalisierungsprogramm (siehe Kapitel 3.3.2.).

${ }^{179}$ Siehe etwa das in Kapitel 3.2.1. besprochene Positionspapier Edirom 2016.

${ }^{180}$ Siehe Kapitel 3.2.3. dieser Arbeit.
} 
die Forschungsdatenverwaltung auch in jene Forschungsgebiete tragen, die vom Digital Turn nur in dem Maße betroffen sind, dass in ihren Projekten zwar computerbasierte Forschungsdaten entstehen, diese letztlich aber immer noch Grundlage analoger Publikationen oder Editionen sind. Hier könnten Werkzeuge, wie sie für das Projektmanagement oder die projektinterne Zusammenarbeit und Aufgabenteilung beispielsweise im Projekt Beethovens Werkstatt eingesetzt werden, ${ }^{181}$ bereits als Prototypen so zur Verfügung gestellt werden, dass die in den Projekten damit erzeugten Daten später standardisiert langfristig archiviert werden können.

Davon völlig unabhängig ist die Notwendigkeit der weiteren Entwicklung einer Digitalen Musikwissenschaft im Sinne der oben in Kapitel 3.1. beschriebenen Teildisziplin. Dies ist notwendig, um den Anschluss musikwissenschaftlicher Forschungen und deren Einbringung in den Kontext und die Fragestellungen der Digital Humanities strukturell zu sichern. Mit dem Musikwissenschaftlichen Seminar Detmold/Paderborn, dem dort eingerichteten Zentrum Musik - Edition - Medien (Zen $|\mathrm{M}| \mathrm{E} \mid \mathrm{M})$ und der Professur für Digitale Musikwissenschaft und Digitale Musikedition sowie den dort anhängigen Forschungsprojekten wie etwa Beethovens Werkstatt existiert dafür ein wichtiger infrastruktureller Baustein im Fach. Die Etablierung weiterer vergleichbarer Institutionen sollte aber durchaus nicht versäumt werden, nicht nur um ein flächendeckendes Problembewusstsein im Fach zu erreichen, sondern letztlich auch um Bedarfe, die sich unabhängig davon ergeben werden, befriedigen zu können. In solchen Zentren könnten institutionalisierte Anlaufstellen für die in den DFG-Richtlinien eingeforderte „Unterstützung und Beratung“ zum Thema Forschungsdatenmanagement eingerichtet werden, die für die technische Umsetzung der Langzeitarchivierung eng mit dem FID Musik zusammenarbeiten sollten.

Auf die allgemeine Fachebene - unabhängig von der Durchführung digitaler Projekte - gehört auch die Diskussion der in den DFG-Leitlinien angesprochenen Frage nach der „Anerkennung der Leistung bei der Verfügbarmachung von Forschungsdaten“ und die in den Grundsätzen zum Umgang mit Forschungsdaten von der Allianz der Wissenschaftsorganisationen ${ }^{182}$ thematisierte Frage nach „den spezifischen Erfordernissen der Disziplinen“ hinsichtlich der Verankerung des professionellen Datenmanagements in „Lehre und Forschung“. Hier hat die Untersuchung in Kapitel 1.1. die wohl größten Defizite gezeigt.

\footnotetext{
${ }^{181}$ Siehe Kapitel 3.2.4. dieser Arbeit.

182 Grundsätze zum Umgang mit Forschungsdaten, verabschiedet von der Allianz der deutschen Wissenschaftsorganisationen am 24. Juni 2010 (https://gfzpublic.gfzpotsdam.de/rest/items/item_2949914_3/component/file_2949913/content).
} 
Die Untersuchungen in allen Kapiteln dieser Arbeit konnten mehr oder weniger nur exemplarisch sein und Schlaglichter auf die wesentlichen Entwicklungen, Voraussetzungen und Bedürfnisse werfen, denen ein modernes Forschungsdatenmanagement begegnen kann oder sollte. Es gibt zahlreiche weitere Editionsprojekte, die (teil-)digital arbeiten und publizieren und dabei Forschungsdaten produzieren, für deren Archivierung wieder ganz andere (und viel zu oft auch noch keine) Lösungen gefunden wurden. Dasselbe gilt für den großen Bereich der musikwissenschaftlichen Quellenerschließungsprojekte. Vor dem Hintergrund der stetig fortschreitenden technischen Entwicklung und des eben postulierten Paradigmas von einer stets deskriptiven Positionsbestimmung, die hinsichtlich des Forschungsdatenmanagements auch immer nur von den Fachvertretern selbst geleistet werden kann und - das gilt es noch hinzuzufügen - freilich dynamisch ist, werden finale Lösungen nie existieren und etabliert werden können. Diese Feststellung mag am Schluss einer solchen Arbeit erst einmal ernüchternd klingen, sollte letztlich vor allem aber als Motivation für alle Beteiligten verstanden werden, diesen Prozess, der das digitale Arbeiten fortan begleiten wird und immer mitzudenken ist, aktiv mitzugestalten. 


\section{Literaturverzeichnis}

Alge 2019

Barbara Alge, Forschungsdatenmanagement in der Musikethnologie, Hildesheim 2019

(Center for World Music - Studies in Music. 3.)

https://doi.org/10.18442/031

Adler 1919

Guido Adler, Methoden der Musikgeschichte, Leipzig 1919

Appel 2015

Bernhard R. Appel und Joachim Veit, Skizzierungsprozesse im Schaffen Beethovens.

Probleme der Erschließung und der Digitalen Edition, in: Die Tonkunst 9 (2015),

S. $122-130$

Bärwald 2016

Manuel Bärwald, Italienische Oper in Leipzig (1744-1756), 2 Bde., Beeskow 2016

(Forum Mitteldeutsche Barockmusik. 6.)

Bärwald/Meyer 2016

Manuel Bärwald und Julia Meyer, Auftakt für Manfred Gorke. Musikhandschriften für die Bach-Forschung im sächsischen Landesdigitalisierungsprogramm, in: BIS. Das Magazin der Bibliotheken in Sachsen 9 (2016), S. 136f.

Blanken 2013

Christine Blanken, Ein wieder zugänglich gemachter Bestand alter Musikalien der Bach-Familie im Verlagsarchiv Breitkopf \& Härtel, in: Bach-Jahrbuch 99 (2013), S. $79-128$

Büttner 2011

Stephan Büttner, Hans-Christoph Hobohm und Lars Müller, Research Data Management, in: Handbuch Forschungsdatenmanagement, hrsg. von Stephan Büttner, Hans-Christoph Hobohm und Lars Müller, Bad Honnef 2011, S. 11-24 
Cox 2015

Susanne Cox, Maja Hartwig und Richard Sänger, Beethovens Werkstatt. Genetische Textkritik und Digitale Musikedition. Projektvorstellung, in: Forum Musikbibliothek 36 (2015), S. 13-20

Eckhardt 2013

Wolfgang Eckhardt, Daniel Fromme, Julia Neumann und Tobias Schwinger, Das DFG-Projekt „,Kompetenzzentrum Forschung und Information Musik“ (KoFIM) an der Staatsbibliothek zu Berlin - Preußischer Kulturbesitz, in: Forum Musikbibliothek 34 (2013), S. 12-21

Eckhardt 2016

Wolfgang Eckhardt, Digitale Dokumentation von Wasserzeichen in Musikhandschriften im Rahmen des Projekts KoFIM, in: Wasserzeichen. Schreiber. Provenienzen. Neue Methoden der Erforschung und Erschließung von Kulturgut im digitalen Zeitalter, hrsg. von Wolfgang Eckhardt, Julia Neumann, Tobias Schwinger und Alexander Staub, Frankfurt/Main 2016 (Sonderbände der Zeitschrift für Bibliothekswesen und Bibliographie. 18.), S. 167-195

Gardner/Springfeld 2014

Matthew Gardner und Sara Springfeld, Musikwissenschaftliches Arbeiten. Eine

Einführung, Kassel 2014 (Bärenreiter Studienbücher Musik. 19.)

Glöckner 1984

Andreas Glöckner, Handschriftliche Musikalien aus den Nachlässen von Carl Gotthelf Gerlach und Gottlob Harrer in den Verlagsangeboten des Hauses Breitkopf 17611769, in: Bach-Jahrbuch 70 (1984), S. 107-116

GMO

Grove Music Online

Grove music program (Oxford University Press), hrsg. von Deane Root (University of Pittsburgh), Philip V. Bohlman (University of Chicago), Jonathan Cross (Christ 
Church, Oxford University), Honey Meconi (University of Rochester), John H.

Roberts (University of California-Berkeley)

www.oxfordmusiconline.com

Götz 2016

Jakob Götz, Musikalisches Repertoire als Spiegel kultureller Identitäten? Einblicke in die Repertoireforschung mit Methoden der Digital Humanities, in: Beitragsarchiv zur Jahrestagung der Gesellschaft für Musikforschung Halle/Saale 2015.

„Musikwissenschaft. Die Teildisziplinen im Dialog“, hrsg. von Wolfgang Auhagen und Wolfgang Hirschmann, Mainz 2017 (urn:nbn:de:101:1-201701063010)

Gray 2009

Jim Gray on eScience. A Transformed Scientific Method, in: The Fourth Paradigm, Data-Intensive Scientific Discovery, hrsg. von Tony Hey, Stewart Tansley und Kristin Tolle, Redmond 2009, S. xvii-xxxi

http://www.immagic.com/eLibrary/ARCHIVES/EBOOKS/M091000H.pdf

Grove

The new Grove dictionary of music and musicians, hrsg. von Stanley Sadie, 20 Bde., London 1985

Grove $^{2}$

The new Grove dictionary of music and musicians. Second edition, hrsg. von Stanley Sadie, 29 Bde., London 2001

Hartwig 2016

Maja Hartwig und Johannes Kepper, Die Spuren des Digitalen. Über die Nachnutzbarkeit digitaler Inhalte, in: „Ei, dem alten Herrn zoll' ich Achtung gern'“. Festschrift für Joachim Veit zum 60. Geburtstag, hrsg. von Kristina Richts und Peter Stadler, München 2016, S. 319-330 
Huth 2010

Karsten Huth, Computerspiele, in: Nestor Handbuch. Eine kleine Enzyklopädie der digitalen Langzeitarchivierung. Version 2.3, hrsg. von Heike Neuroth, Achim Oßwald, Regine Scheffel, Stefan Strathmann und Mathias Jehn, Boizenburg 2010, Kap. 17:116-17:130

Kepper 2011

Johannes Kepper, Musikedition im Zeichen neuer Medien. Historische Entwicklung und gegenwärtige Perspektiven musikalischer Gesamtausgaben, Norderstedt 2011 (Schriften des Instituts für Dokumentologie und Editorik. 5.)

Kepper 2014

Johannes Kepper, Solveig Schreiter und Joachim Veit, Freischütz analog oder digital. Editionsformen im Spannungsfeld von Wissenschaft und Praxis, in: Editio. Internationales Jahrbuch für Editionswissenschaft 28 (2014), S. 127-150

Kossek 2012

Brigitte Kossek, Einleitung. Digital turn?, in: Digital Turn? Zum Einfluss digitaler Medien auf Wissensgenerierungsprozesse von Studierenden und Hochschullehrenden, hrsg. von Markus F. Peschl und Brigitte Kossek, Göttingen 2012, S. 7-19

Loos 2012

Helmut Loos, Gilbert Stöck und Nicole Waitz, Untersuchungen zum Repertoire des Leipziger Thomanerchores zwischen 1808 und 2008 auf der Grundlage EDVgestützter statistischer Erhebungen, in: 800 Jahre Thomana. Glauben - Singen Lernen, hrsg. von Stefan Altner und Marin Petzoldt, Leipzig 2012, S. 218-233

Maul/Wollny 2003

Michael Maul und Peter Wollny, Quellenkundliches zu Bach-Aufführungen in Köthen, Ronneburg und Leipzig zwischen 1720 und 1760, in: Bach-Jahrbuch 89 (2003), S. 97141 
MGG

Die Musik in Geschichte und Gegenwart. Allgemeine Enzyklopädie der Musik, hrsg. von Friedrich Blume, 17 Bde., Kassel 1951-1986

$\mathrm{MGG}^{2}$

Die Musik in Geschichte und Gegenwart. Allgemeine Enzyklopädie der Musik. Zweite neubearbeitete Ausgabe, hrsg. von Ludwig Finscher, 29 Bde., Kassel und Stuttgart 1994-2008

MGG Online

MGG Online. Die Musik in Geschichte und Gegenwart, hrsg. von Laurenz Lütteken, Kassel (Bärenreiter-Verlag Karl Vötterle GmbH \& Co. KG), Stuttgart (J.B. Metzler, Part of Springer Nature, Springer Verlag GmbH) und New York (Répertoire International de Littérature Musicale Inc.)

https://mgg-online.com

Müller 2011

Lars Müller, An der Schwelle zum Vierten Paradigma. Datenmanagement in der Klimaplattform, in: Konferenz Digitale Wissenschaft. Stand der Entwicklung digital vernetzter Forschung in Deutschland, hrsg. von Silke Schomburg, Claus Leggewie, Henning Lobin und Cornelius Puschmann, zweite, ergänzte Fassung, Köln 2011, S. $131-137$ http://fiz1.fh-potsdam.de/volltext/DigiWis/13469.pdf

Müller 2015

Kai Müller, Innerdisziplinäre Interdisziplinarität. Ein Gespräch mit Wolfgang Auhagen, in: Neue Zeitschrift für Musik (6/2015), S. 8-11

Nägele 2016

Rainer Nägele, Digitale historische Musikwissenschaft. Eine fragwürdige Disziplin, in: kontrovers. musiconn.blog vom 14. Januar 2016 https://kontrovers.musiconn.de/2016/01/14/digitale-historische-musikwissenschafteine-fragwuerdige-disziplin 
Neumann 2016

Julia Neumann, Erschließung und Retrieval von Provenienzen und Provenienzmerkmalen in Musikhandschriften. Aktueller Stand und Perspektiven, in:

Wasserzeichen. Schreiber. Provenienzen. Neue Methoden der Erforschung und Erschließung von Kulturgut im digitalen Zeitalter, hrsg. von Wolfgang Eckhardt, Julia Neumann, Tobias Schwinger und Alexander Staub, Frankfurt/Main 2016 (Sonderbände der Zeitschrift für Bibliothekswesen und Bibliographie. 18.), S. 69-93

Positionspapier Edirom 2016

Nikolaos Beer, Kristin Herold, Peter Stadler und Joachim Veit, Künftige Rahmenbedingungen musikwissenschaftlicher Editionsprojekte aus der Sicht des Virtuellen Forschungsverbunds Edirom (ViFE) am Musikwissenschaftichen Seminar Detmold/Paderborn, April 2016 http://www.edirom.de/files/Positionspapier_ViFE_2016-04.pdf

Rebmann 2016

Martina Rebmann, Musikwissenschaftliche Grundlagenforschung. Das Pilotprojekt „, Kompetenzzentrum Forschung und Information Musik“ (KoFIM) an der Staatsbibliothek zu Berlin, in: Wasserzeichen. Schreiber. Provenienzen. Neue Methoden der Erforschung und Erschließung von Kulturgut im digitalen Zeitalter, hrsg. von Wolfgang Eckhardt, Julia Neumann, Tobias Schwinger und Alexander Staub, Frankfurt/Main 2016 (Sonderbände der Zeitschrift für Bibliothekswesen und Bibliographie. 18.), S. 9-17

Reipsch 2001

Brit Reipsch, „, Damit er von einem Meister, und nicht von allerley Pfuschern bestände“. Neue Überlegungen zu Manuskripten Johann Caspar Dietels, in: Bach und seine mitteldeutschen Zeitgenossen. Bericht über das Internationale Musikwissenschaftliche Kolloquium. Erfurt und Arnstadt 13. bis 16. Januar 2000, hrsg. von Rainer Kaiser, Eisenach 2001 (Schriften zur mitteldeutschen Musikgeschichte. 4.), S. 161-178 
Rümpel 2011

Stefanie Rümpel, Der Lebenszyklus von Forschungsdaten, in: Handbuch

Forschungsdatenmanagement, hrsg. von Stephan Büttner, Hans-Christoph Hobohm und Lars Müller, Bad Honnef 2011, S. 25-34

Schmale 2016

Wolfgang Schmale, Digital Musicology im Kontext der Digital Humanities, in:

Wissenskulturen der Musikwissenschaft. Generationen - Netzwerke - Denkstrukturen, hrsg. von Sebastian Bolz, Moritz Kelber, Ina Knoth und Anna Langenbruch, Bielefeld 2016, S. 299-310

Schmidt 2015

Dörte Schmidt, Neue philologische Strategien in der Musikwissenschaft und die Politiken der Forschung, in: Tagesspiegel Online vom 4. November 2015 http://www.tagesspiegel.de/themen/digital-humanities/prof-dr-doerte-schmidt-neuephilologische-strategien-in-der-musikwissenschaft-und-die-politiken-derforschung/12541310.html

Schulze 1977

Hans-Joachim Schulze, Katalog der Sammlung Manfred Gorke. Bachiana und andere Handschriften und Drucke des 18. und frühen 19. Jahrhunderts, Leipzig 1977 (Bibliographische Veröffentlichungen der Musikbibliothek der Stadt Leipzig)

Schwindt-Gross 1992

Nicole Schwindt-Gross, Musikwissenschaftliches Arbeiten. Hilfsmittel, Techniken, Aufgaben, Kassel 1992 (Bärenreiter Studienbücher Musik. 2.)

Schwinger 2016

Tobias Schwinger, Digitale Dokumentation von Autographen und Abschriften im Rahmen des Projekts KoFIM, in: Wasserzeichen. Schreiber. Provenienzen. Neue Methoden der Erforschung und Erschließung von Kulturgut im digitalen Zeitalter, hrsg. von Wolfgang Eckhardt, Julia Neumann, Tobias Schwinger und Alexander 
Staub, Frankfurt/Main 2016 (Sonderbände der Zeitschrift für Bibliothekswesen und Bibliographie. 18.), S. 121-133

Stöck 2012

Gilbert Stöck und Peter Scholz, Das Online-Portal zur Repertoire-Erforschung des Thomanerchores Fragestellungen, Methoden und Zielsetzungen, in: Forum Musikbibliothek 33 (2012), S. 34-39

\section{Veit 2013}

Joachim Veit, Freischütz Digital. Projekteinblick, in: Forschungsinfrastrukturen für die Geistes- und Sozialwissenschaften, hrsg. vom Bundesministerium für Bildung und Forschung, Bonn 2013, S. 31

https://www.kowi.de/Portaldata/2/Resources/fp/Forschungsinfrastrukturen-Geistesund-Sozialwissenschaften.pdf

Wünsche 2018

Stephan Wünsche, Forschungsdaten in musikwissenschaftlichen und musikpädagogischen Dissertationen. Eine Stichprobe anhand der im Jahr 2015 in Deutschland angenommenen Arbeiten, Berlin 2018 (Berliner Handreichungen zur Bibliotheks- und Informationswissenschaft. 433.) http://dx.doi.org/10.18452/19457 